\title{
Traces in braided categories
}

\author{
D. Gurevich, R. Leclercq \\ ISTV, Université de Valenciennes, 59304 Valenciennes, France \\ P. Saponov, \\ Theory Department, Institute for High Energy Physics, 142284 Protvino, Russia
}

November 7, 2018

\begin{abstract}
With any even Hecke symmetry $R$ (that is a Hecke type solution of the Yang-Baxter equation) we associate a quasitensor category. We formulate a condition on $R$ implying that the constructed category is rigid and its commutativity isomorphisms $R_{U, V}$ are natural in the sense of [T]. We show that this condition leads to rescaling the initial Hecke symmetry. We suggest a new way of introducing traces as properly normalized categorical morphisms End $(V) \rightarrow \mathbb{K}$ and deduce the corresponding normalization from categorical dimensions.
\end{abstract}

AMS Mathematics Subject Classification, 1991 : 18D10, 81R50

Key words : quasitensor (braided) rigid category, Hecke algebra, categorical dimension, trace

\section{Introduction}

The main purpose of this paper is to introduce some braided categories arising from "nonquasiclassical" Hecke symmetries constructed in [G] and to suggest a way of defining categorical traces

$$
\operatorname{tr}=\operatorname{tr}_{V}: \text { End }(V) \rightarrow \mathbb{K}, V \in \mathrm{Ob}(\mathcal{C}) .
$$

in a somewhat elementary way without using any ribbon element (see below). Hereafter $\mathrm{Ob}(\mathcal{C})$ stands for the set of objects of the category $\mathcal{C}$ and $\mathbb{K}$ stands for the basic field, always $\mathbb{C}$ or $\mathbb{R}$.

Let us precise from the very beginning that by braided categories we mean monoidal tensor or quasitensor ones whose objects are vector spaces with the usual tensor product and whose natural associativity isomorphisms are identical (for the terminology, cf. for example [CP, T]). Thus, the structure of such a category is principally determined by commutativity isomorphisms

$$
R_{U, V}: U \otimes V \rightarrow V \otimes U, \quad U, V \in \mathrm{Ob}(\mathcal{C}) .
$$

These isomorphisms are assumed to satisfy the so-called Yang-Baxter (YB) equation. They play the role of the usual flip which transposes the factors. We call the isomorphisms (1.2) YB operator, braiding, or quantum $R$-matrix.

Moreover, we assume them to be natural in the sense of [T]. This means that for any two categorical morphisms $f: U \rightarrow U^{\prime}$ and $g: V \rightarrow V^{\prime}$ one has:

$$
(g \otimes f) \circ R_{U, V}=R_{U^{\prime}, V^{\prime}} \circ(f \otimes g) .
$$

We show that this condition leads to a normalization of the commutativity isomorphisms different from that (1.8) usually employed for Hecke type braidings. (Let us note that even in the case of the quantum group $U_{q}(\operatorname{sl}(n))$ the YB operators coming from the universal R-matrix must be rescaled.) 
In what follows we identify $\mathbb{K}$ and the End $(I)$ where $I$ is the identity object in the sense of [CP]. Moreover, we use the notation $\operatorname{End}(V)=\operatorname{Hom}(V, V)$ for the objects of the category which sometimes are called internal (endo)morphisms (cf. [DM]). By contrast, the morphisms of the category in question will be called "categorical morphisms". For example, categorical morphisms of the category $\mathbf{g}-$ Mod of modules over a Lie algebra $\mathbf{g}$ are elements of Hom $(U, V)$, $U, V \in \mathrm{Ob}(\mathrm{g}-\mathrm{Mod})$, commuting with the action of $\mathbf{g}$.

Initially, categories equipped with commutativity morphisms were introduced by S. MacLane McL1]. However, he only considered involutary YB operators $\left(R^{2}=\mathrm{id}\right)$ called in what follows "symmetries". A new wave of interest in braided categories (but with noninvolutary braidings) arose in connection with integrable system theory. More precisely, such categories appeared as those of modules of Drinfeld-Jimbo quantum groups (QG) $U_{q}(\mathbf{g})$ playing an important role in this theory (we will denote this category $U_{q}(\mathbf{g})-\mathrm{Mod}$ ). Besides, the QG have found many other interesting applications, in particular, in noncommutative geometry.

The problem we consider in the paper is a categorical definition of the trace (1.1). What is a reasonable generalization of the basic property of the classical trace

$$
\operatorname{tr}[X, Y]=0, \quad \forall X, Y \in \operatorname{End}(V)
$$

where $[X, Y]$ is the commutator of two endomorphisms円?

If the braiding in a given braided category is involutary and invertible by column in the sense of the formula (2.48) then there exists a natural generalization of the above commutator such that relation (1.4) is still valid for this commutator and an appropriate trace. For example it is so for a super-commutator and super-trace defined in a super-category. Other examples can be found in [G]

Let us note that the definition of the trace (1.1) in a category equipped with a symmetry makes use of this symmetry and of the identification $\operatorname{End}(V)=V \otimes V^{*}$ where $V^{*}$ is an object dual to $V$ (all categories in question are assumed to be rigid, i.e. for any object of the category its dual is also an object). Up to our knowledge for the first time such a trace has been introduced in $\mathrm{DP}]$.

Once such a trace is defined one can introduce a categorical dimension $\operatorname{dim}(V)$ of an object $V$ by setting $\operatorname{dim}(V)=\operatorname{tr}(\mathrm{id})$. It is easy to see that

$$
\operatorname{dim}(U \oplus V)=\operatorname{dim}(U)+\operatorname{dim}(V), \operatorname{dim}(U \otimes V)=\operatorname{dim}(U) \operatorname{dim}(V),
$$

i.e. the dimension can be considered as an additive and multiplicative (a-m) functional on the objects of the category.

However, a direct application of the approach of [DP] to the category $U_{q}(\mathbf{g})-$ Mod gives "dimension" which is not an a-m functional. This is the reason why one has to introduce a correction in the definition of "dimensions" turning them into an a-m functional. The correction is connected to the so-called ribbon element in the corresponding QG. The image of this element is sometimes called "twist" (cf. [T]). The categories possessing a braiding and a twist are called ribbon. The trace in such categories is defined via some combination of the ribbon element and another element $u$ due to Drinfeld (cf. $[\mathrm{CP}$ ) and this leads to the dimension which is an a-m functional.

\footnotetext{
${ }^{1}$ Sometimes this property is attributed to the quantum trace (cf. H]). Equivalent form of this property $\operatorname{tr}\left(g X g^{-1}\right)=\operatorname{tr} X(g \in$ End $(V)$ is assumed to be invertible) appeared in $[\Gamma]$. However, all this is true if we assume $X, Y$ and $g$ to be categorical morphisms. But in this case the conditions above become meaningless. It is easy to see by taking a simple object $V$, since it only admits scalar categorical morphisms and these conditions degenerate.

${ }^{2}$ In this case the trace can be treated in termes of "R-cyclic cohomology" which can be naturally defined via the operator $R$ (in the spirit of super-cyclic cohomology). However, apparently there does not exist any reasonable similar treatment of the trace in the categories under consideration.
} 
Some generalization of this construction of the trace has been suggested in [BW]. Suggested in that paper is a way to define the notion of dimension in some categories without any braiding. Such a category is introduced as that of $H$-modules where $H$ is a Hopf algebra. Instead of a universal quantum $R$-matrix (i.e. the element giving rise to a braiding) the authors of [BW] use another element allowing to identify any object $V$ with its second dual $V^{* *}$.

In the present paper we restrict ourselves to categories equipped with braidings and suggest another somewhat elementary way of introducing traces.

Before discussing this way let us introduce some notations and definitions.

A Yang-Baxter operator

$$
R: V^{\otimes 2} \rightarrow V^{\otimes 2}
$$

will be called a Hecke symmetry if in addition to the Yang-Baxter equation

$$
R_{12} R_{23} R_{12}=R_{23} R_{12} R_{23}
$$

it also satisfies the relation

$$
(q \mathrm{id}-R)\left(q^{-1} \mathrm{id}+R\right)=0, \quad q \in \mathbb{K} .
$$

Here the standard tensor notations have been used for the equations and operators in tensor product of spaces. Equation (1.7) is written in $V^{\otimes 3}$ and one assumes

$$
R_{12}=R \otimes \mathrm{id}, \quad R_{23}=\mathrm{id} \otimes R,
$$

where id is the identity operator on $V$.

The nonzero parameter $q$ is taken to be generic, which means it is not a root of unity: $\forall k \in \mathbb{N}, k \neq 1, q^{k} \neq 1$. As a consequence none of the so-called $q$-numbers $k_{q}$ is equal to zero

$$
k_{q} \equiv \frac{q^{k}-q^{-k}}{q-q^{-1}} \neq 0 \quad \forall k \in \mathbb{N} .
$$

Let us assign to such a Hecke symmetry "symmetric" $\Lambda_{+}(V)$ and "skew-symmetric" $\Lambda_{-}(V)$ algebras by

$$
\Lambda_{+}(V)=T(V) /\{\operatorname{Im}(q \text { id }-R)\}, \quad \Lambda_{-}(V)=T(V) /\left\{\operatorname{Im}\left(q^{-1} \mathrm{id}+R\right)\right\} .
$$

Here $T(V)$ stands for the free tensor algebra of the space $V$ and $\{I\}$ denotes the ideal generated by a subset $I$ in a given algebra. Let $\Lambda_{ \pm}^{l}(V)$ be a homogeneous component of the algebra $\Lambda_{ \pm}(V)$ of the degree $l$. We call a Hecke symmetry even if there exists an integer $p$ such that the component $\Lambda_{-}^{l}(V)$ is trivial at $l>p$ and it is one-dimensional at $l=p$. The integer $p$ will be called a rank of $V$ and be denoted $\mathrm{rk}(V)$.

In the latter 80's one of the authors (D.G.) constructed examples of Hecke type braidings which differ drastically from those related to the QG (see $[\bar{G}]$ and references therein). Namely, it was shown that there exist a lot of Hecke symmetries $R$ such that $n=\operatorname{dim}(V)>p=\operatorname{rk}(V)$. Here by $\operatorname{dim}(V)$ we mean the usual dimension of the space $V$. Note that these Hecke symmetries

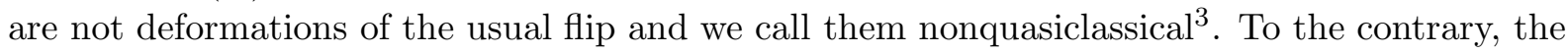
Hecke symmetry coming from the QG $U_{q}(s l(n))$ is a deformation of the flip and we call it quasiclassical.

In this paper we introduce a braided category generated by a vector space $V$ equipped with a Hecke symmetry such that its braidings are natural. We call it the Schur-Weyl (SW) category and denote as $\mathrm{SW}(\mathrm{V})$. Let us remark that similar categories were considered in some papers (cf.

\footnotetext{
${ }^{3}$ Some of them have been independently introduced in [DL].
} 
[KW], [H], [B] ). However, if the authors of [KW] "reconstruct" an existing category we have no category at the very beginning and should first construct it.

We construct the category directly by giving the list of objects and categorical morphisms without using any RTT algebra habitually employed for that ([襾, [B] $)$. This leads to different defining morphisms of the category and finally, to the condition (2.33) ensuring naturality of the braidings $R_{U, V}$. (In a separate publication one of us (R.L.) shows that in the class of nonquasiclassical Hecke symmetries from [G] there exists a big subclass of those satisfying this condition.)

Now let us describe the category under consideration. Any object of such a category is a direct sum of simple ones and in this sense the category $\mathrm{SW}(\mathrm{V})$ is spanned by simple (basic) objects. These basic objects are labelled by partitions (or what is the same by Young diagrams)

$$
\lambda=\left(\lambda_{1}, \lambda_{2}, \ldots, \lambda_{p-1}\right), \quad \lambda_{i+1} \leq \lambda_{i},
$$

$\lambda_{i}$ being nonnegative integers. In the sequel we will use the notation $\lambda \vdash k$ for each $\lambda$ being a partition of the integer $k$ that is $\sum_{i} \lambda_{i}=k$. The number of nonzero components of a partition $\lambda$ is called its height and will be denoted $\ell(\lambda)$. For an object corresponding to a diagram $\lambda$ we introduce the notation $V_{\lambda}$.

Let us stress that the tensor product of two basic objects is a direct sum of basic ones. This naturally leads us to the notion of Grothendieck semiring (and hence ring) of the category which turns out to be the same as for the categories of $\operatorname{sl}(n)$ - (or $U_{q}(s l(n))$ )-modules. The only difference is that the role of the classical dimension $n=\operatorname{dim}(V)$ is played by the rank $p=\operatorname{rk}(V)$. Thus, we can consider the dimensions of objects of the category as a functional on the Grothendieck semiring. Since it is an a-m functional we come to the problem of describing the whole family of a-m functionals and select those related to traces.

By using the standard technique of symmetric functions (cf. [M]) we show in Proposition 15 that such a functional $f$ is determined by its values on objects $V_{\left(1^{k}\right)}, k=1,2, \ldots, p-1$, and its value on a basic object $V_{\lambda}$ is equal to

$$
f\left(V_{\lambda}\right)=s_{\lambda}\left(\alpha_{1}, \alpha_{2}, \ldots, \alpha_{p}\right)
$$

where $s_{\lambda}$ is the Schur function (polynomial) in $p$ variables corresponding to the partition $\lambda$. The numbers $\left(-\alpha_{i}\right)$ are the roots of the polynomial

$$
\phi(t)=t^{p}+f_{1} t^{p-1}+\ldots+f_{p-1} t+1, \quad f_{k}=f\left(V_{\left(1^{k}\right)}\right) .
$$

(Note, that the classical (usual) dimension is also an a-m functional. Therefore, the above result allows one to calculate the classical dimension of spaces $V_{\lambda}$ provided that the Poincaré series $P_{-}(t)$ (see Remark 19) is known (for involutary symmetries such a calculation has been done in $[\mathrm{GM}]$ ). Remark, that if a Hecke symmetry $R(q)(1.6)$ is a deformation of an involutary one $R(1)$ then the usual dimension of corresponding spaces $V_{\lambda}$ will be the same in both cases. Indeed, being an integer number such a dimension is stable under deformation, while the categorical dimension is a function in $q$ which becomes integer at $q=1$.)

Having classified all a-m functionals one can put the question: which a-m functionals come from SW categories? More precisely, for which a-m functional on a given Grothendieck semiring (with a fixed $p$ ) there exists a SW category such that the categorical dimension of its objects coincides with this functional?

Now we are going back to the problem of defining traces on objects End $(U)=U \otimes U^{*}$ (for the sake of concreteness we consider algebras of left endomorphisms). Let us assume that trace is defined on End $(U \oplus V)=(U \oplus V) \otimes\left(U^{*} \oplus V^{*}\right)$ via

$$
\left.\operatorname{tr}\right|_{\operatorname{End}(U) \oplus \operatorname{End}(V)}=\left.\operatorname{tr}\right|_{\operatorname{End}(U)}+\left.\operatorname{tr}\right|_{\operatorname{End}(V)}
$$


being extended to $U \otimes V^{*} \oplus V \otimes U^{*}$ by 0 . Then traces are completely defined by their values on simple objects. If $V$ is such an object then the trivial component in the product $V \otimes V^{*}$ is unique. So, the morphism (1.1) for such $V$ (being nothing but a multiple of the projection on the trivial component) is uniquely defined up to a nontrivial factor.

As a trace we take this properly normalized morphism. Our "proper" normalization is fixed by the requirement that the corresponding dimension should be an a-m functional on the corresponding Grothendieck semiring. Thus, this normalization is a collective phenomenon.

Remark that our method to define traces as properly normalized categorical morphisms is valid, in principle, for any category for which the Grothendieck semiring is well-defined. This method does not make use of either ribbon element or twist. It is sometimes useful to employ twists in order to calculate categorical dimensions (as it is done in [H]) but it is not reasonable to introduce it in the definition of traces. Also, it turns out that this method is useful for studying K-theory of some algebras related to SW categories and computing noncommutative index for them. This application will be presented elsewhere.

As for the category under consideration a computation shows that the corresponding dimensions of basic objects are

$$
\operatorname{dim}_{q}\left(V_{\lambda}\right)=s_{\lambda}\left(q^{p-1}, q^{p-3}, \ldots, q^{-(p-1)}\right) .
$$

In a little bit different form the formula (1.12) was also given in [KW] and [ [ ] ]. (Also remark that in the case of the category $U_{q}(s l(n))$ - Mod the above formula is equivalent to that from Go .)

However, behind this nondetailed information about the objects encoded in their dimensions different and rich structures are hidden. For example, traces (1.1) in the categories in question are defined via some matrix $C$ entering the formula for the trace on the basic space $V$ and extended in a proper way onto the whole category (the extension of $C$ matrix to the space $V_{\lambda}$ will be denoted $C_{\lambda}$ ). It is worth mentioning that the matrices $C_{\lambda}$ are essentially different in quasiclassical and nonquasiclassical cases while the categorical dimensions of objects which are nothing but the properly normalized usual traces of $C_{\lambda}$ depend only on $\lambda$ and $p=\operatorname{rk}(V)$ via the formula (1.12).

The paper is organized as follows. In Section 2 we construct all the necessary elements of a Schur-Weyl category: the class of objects, categorical morphisms, the quasitensor and rigid structure. At the end of Section 2 we discuss the role of the condition (2.33) for applications. In Section 3 we describe the full set of a-m functionals on the objects of $\mathrm{SW}(\mathrm{V})$, define the trace in $\operatorname{End}(\mathrm{U}), \forall U \in \mathrm{Ob}(\mathrm{SW}(\mathrm{V}))$, and calculate $\operatorname{dim}_{q} U$ as the value of trace on identity morphism in $\operatorname{End}(\mathrm{U})$.

Acknowledgement Two of the authors (D.G. and P.S) would like to thank Max-PlanckInstitut für Mathematik (Bonn) where the paper was completed for warm hospitality.

\section{Schur-Weyl category generated by a Hecke symmetry}

In this section we describe the construction of Schur-Weyl category $\mathrm{SW}(\mathrm{V})$ (see Introduction) generated by a finite dimensional vector space $V$ equipped with a Hecke symmetry. We supply the category with attributes of that $U_{q}(s l(n))$-Mod. In particular, all categories $\mathrm{SW}(\mathrm{V})$ are $\mathbb{K}$-linear rigid quasitensor ones (for precise definitions see [CP, McL2]). As was shortly outlined in Introduction, the basic objects of $\mathrm{SW}(\mathrm{V})$ are some linear subspaces $V_{\lambda}$ of the tensor algebra $T(V)$, while the categorical morphisms will be specific linear mappings of the objects (see below). Consequently, a $\mathbb{K}$-linear structure of such a category is obvious: as the direct sum of objects we will take the usual direct sum of linear spaces and the trivial (zero) vector space will be the 
null object of the category. So, we should only define the structure of monoidal category and fix braiding isomorphisms converting the monoidal structure into the quasitensor (or braided) one. Besides, to have a rigid category, we must ensure that the dual space $V^{*}$ of an arbitrary $V \in \mathrm{Ob}(\mathrm{SW}(\mathrm{V}))$ is also an object of the category. Now we pass to the explicit construction of the mentioned components of the category SW(V) taking into account condition (1.3).

\subsection{Class of objects}

Let us fix some finite dimensional vector space $V$, $\operatorname{dim} V=n$, and choose a basis $\left\{e_{i}\right\}$ in this space. Consider a linear operator $R(1.6)$ whose action on the basis elements reads:

$$
R\left(e_{i} \otimes e_{j}\right)=e_{r} \otimes e_{s} R_{i j}^{r s},
$$

where the summation over the repeated indices is understood. In what follows we assume $R$ to be a Hecke symmetry with a generic $q \in \mathbb{K}$.

Besides, we will suppose $R$ to be an even symmetry of a rank $p \leq n=\operatorname{dim} V$. This means that the Poincaré series $P_{-}(t)$ related to the "skew-symmetric" algebra $\wedge_{-}(V)(1.10)$ is a monic polynomial of $p$-th degree, that is a polynomial in $t$ whose leading coefficient is equal to 1 .

Throughout the paper we will use the compact notation for the relations including matrices and vector spaces. In such notations the indices will stand for a number of space rather than for a particular matrix or vector component. A basis vector $e_{i}$ in the $k$-th matrix space is denoted as $e_{\langle k|}$, while for the basis vector of dual space we will write $e^{|k\rangle}$. The same principle is applied to components of arbitrary tensors.

For example, the above relation will look liket

$$
R\left(e_{\langle 1|} \otimes e_{\langle 2|}\right):=e_{\langle 1|} \otimes e_{\langle 2|} R_{\langle 12|}^{|12\rangle}:=e_{\langle 1|} \otimes e_{\langle 2|} R_{12}
$$

In this notation the summation over the repeated indices is represented by an expression which contains the same upper and lower case indices with properly oriented brackets as shown in the examples below:

$$
e_{\langle 1|} \otimes e^{|1\rangle}:=\sum_{i} e_{i} \otimes e^{i}, \quad e_{\langle 1|} \cdot T_{1}:=e_{\langle 1|} T_{\langle 1|}^{|1\rangle}:=\sum_{j} e_{j} T_{i}^{j}, \quad M_{1} R_{12} N_{2}:=\sum_{a, b} M_{a}^{i_{1}} R_{j_{1} b}^{a i_{2}} N_{j_{2}}^{b}
$$

but

$$
e^{|1\rangle} \otimes e_{\langle 1|}:=e^{i} \otimes e_{j}, \quad e_{\langle 1|} \otimes e^{|2\rangle}:=e_{i} \otimes e^{j}, \quad T_{1} \cdot e_{\langle 1|}:=T_{j}^{i} e_{k}
$$

and so on.

Now we use the fact, that in each homogeneous component $V^{\otimes m}$ of the tensor algebra $T(V)$ one can realize the so called local representation of the Hecke algebra $H_{m}$ [DJ] via a given Hecke symmetry冋.

The Hecke algebra $H_{m}$ is generated by the unit element $\mathrm{id}_{H}$ and $m-1$ generators $\sigma_{k}$ subject to the following relations:

$$
\left.\begin{array}{l}
\sigma_{i} \sigma_{i+1} \sigma_{i}=\sigma_{i+1} \sigma_{i} \sigma_{i+1} \\
\sigma_{i} \sigma_{j}=\sigma_{j} \sigma_{i} \quad \text { if }|i-j| \geq 2 \\
\left(\sigma_{i}-q \operatorname{id}_{H}\right)\left(\sigma_{i}+q^{-1} \operatorname{id}_{H}\right)=0
\end{array}\right\} i=1,2, \ldots m-1
$$

The local representation of $H_{m}$ in $V^{\otimes m}$ is of the form:

$$
\sigma_{i} \rightarrow \rho_{R}\left(\sigma_{i}\right) \equiv R_{i i+1}=\mathrm{id}_{i-1} \otimes R \otimes \mathrm{id}_{m-i-1} \in \operatorname{End}\left(V^{\otimes m}\right) .
$$

\footnotetext{
${ }^{4}$ The symbol := means "by definition".

${ }^{5}$ For the review on Hecke algebra the reader is referred to the recent work $\mathrm{OP}$
} 
In the further construction of $\mathrm{SW}(\mathrm{V})$ category the central role belongs to the fact that the Hecke algebra $H_{m}$ for a generic $q$ is semisimple and can be decomposed into a direct sum of ideals. Moreover, since the Hecke algebra $H_{m}$ at a generic $q$ is isomorphic to the group algebra $\mathbb{K}\left[S_{m}\right]$ of the $m$-th order permutation group $S_{m}$, its primitive idempotents generating the ideals in mentioned decomposition can be put into one-to-one correspondence with the set of all standard Young tableaux connected with each possible partition $\lambda$ of the integer $m$. Speaking more explicitly, given the algebra $H_{m}$ and a partition $\lambda \vdash m$, one can construct some polynomials $Y_{i i}^{\lambda}$ in generators $\left\{\sigma_{i}\right\}$ which turn out to be the primitive idempotents of Hecke algebra $H_{m}$ (for detailed description of such a construction see review [OP]):

$$
\begin{gathered}
\operatorname{id}_{H}=\sum_{\lambda \vdash m} \sum_{i=1}^{d_{\lambda}} Y_{i i}^{\lambda}, \\
Y_{i i}^{\lambda} Y_{j j}^{\mu}=\delta_{i j} \delta^{\lambda \mu} Y_{i i}^{\lambda} .
\end{gathered}
$$

In the above formulas the index $i$ runs from 1 to $d_{\lambda} \equiv \operatorname{dim} \lambda$, that is to the number of all standard Young tableaux, corresponding to the Young diagram of the partition $\lambda \vdash m$. Recall, that a Young tableau is called standard if it is filled with successive integers from 1 to $m$ in such a way that the integers increase from left to right in each row and from top to bottom in each column. The standard Young tableaux (and hence the primitive idempotents $Y_{i i}^{\lambda}$ ) may be lexicographically ordered in many ways and for definiteness we fix an order in which the first idempotent $Y_{11}^{\lambda}$ corresponds to the Young tableau filled by integers consequently increasing by 1 when going down in each column. Here is an example for the Young tableau corresponding to the partition $\lambda=\left(3,2^{2}, 1\right)$ :

$$
Y_{11}^{\lambda} \longleftrightarrow \begin{array}{|l|l|l|}
\hline 1 & 5 & 8 \\
\hline 2 & 6 & \\
\cline { 1 - 2 } 3 & 7 & \\
\cline { 1 - 1 } 4 & \multicolumn{2}{|l}{} \\
\cline { 1 - 1 } &
\end{array}
$$

A primitive idempotent corresponding to the partition $\lambda=\left(1^{m}\right)$ will be called the antisymmetrizer and be denoted as $A^{(m)}$. There is one important circumstance about the images of $A^{(m)}$ in $\operatorname{End}\left(V^{\otimes m}\right)$ with respect to representation (2.1). Since the YB operator $R$ is taken to be an even Hecke symmetry of rank $p$, the image of the $p$-th order antisymmetrizer

$$
\mathcal{A}^{(p)}:=\rho_{R}\left(A^{(p)}\right): V^{\otimes p} \rightarrow V^{\otimes p}
$$

is one dimensional. So, the action of this projector on an arbitrary basis element of $V^{\otimes p}$ can be presented in the form:

$$
\mathcal{A}^{(p)} e_{\langle 1|} \otimes \ldots \otimes e_{\langle p|}=e_{\langle 1|} \otimes \ldots \otimes e_{\langle p|} v^{|1 \ldots p\rangle} u_{\langle 1 \ldots p|},
$$

where components of the tensors $u$ and $v$ belong to the field $\mathbb{K}$. Due to (2.3) the normalization of $u$ and $v$ is fixed to be $u_{\langle 1 \ldots p|} v^{|1 \ldots p\rangle}=1$. The images of antisymmetrizers $A^{(m)}$ with $m>p$ are trivial:

$$
\operatorname{Im} \rho_{R}\left(A^{(m)}\right)=0 \quad \forall m \geq p+1 .
$$

In accordance with general theory each primitive idempotent $Y_{i i}^{\lambda}$ generates a left or right ideal of the Hecke algebra $H_{m}$ by means of left or right multiplications on all possible elements of $H_{m}$. Equivalently, one can consider the left or right regular module over $H_{m}$ which as a vector space is $H_{m}$ itself and left or right action consists in the left or right multiplication by Hecke algebra elements. In these terms the left or right ideals generated by primitive idempotents will be irreducible left or right $H_{m}$ submodules. Let us denote left and right submodules generated 
by $Y_{i i}^{\lambda}$ as $M_{\lambda}^{l}(i)$ and $M_{\lambda}^{r}(i)$ respectively. As a direct consequence of (2.2) one can write the following decomposition:

$$
H_{m}=\bigoplus_{\lambda \vdash m} \bigoplus_{i=1}^{d_{\lambda}} M_{\lambda}^{r}(i),
$$

and a similar decomposition holds for left submodules $M_{\lambda}^{l}(i)$.

Consider in more detail the structure of the component of (2.7) corresponding to a fixed partition $\lambda \vdash m$. Let us denote

$$
\bar{M}_{\lambda} \equiv \bigoplus_{i=1}^{d_{\lambda}} M_{\lambda}^{r}(i) \equiv \bigoplus_{i=1}^{d_{\lambda}} M_{\lambda}^{l}(i) .
$$

The set $\bar{M}_{\lambda}$ is a two sided submodule in $H_{m}$. Let us dwell upon the question of linear basis in $\bar{M}_{\lambda}$. As is known from the theory of Hecke algebra, $\bar{M}_{\lambda}$ is isomorphic to the algebra $M a t_{d_{\lambda}}(\mathbb{K})$ of $d_{\lambda} \times d_{\lambda}$ matrices. Therefore in $\bar{M}_{\lambda}$ one can find the set of $d_{\lambda}^{2}$ linear independent quantities $Y_{i j}^{\lambda}$, which are some polynomials in generators $\sigma_{i}$ of $H_{m}$ obtained from the primitive idempotents $Y_{i i}^{\lambda}$. These quantities form a linear basis in $\bar{M}_{\lambda}$ and obey the algebra of matrix units $E_{i j}$ which constitute the linear basis in $\operatorname{Mat}_{d_{\lambda}}(\mathbb{K})$ :

$$
Y_{i j}^{\lambda} Y_{k l}^{\lambda}=\delta_{j k} Y_{i l}^{\lambda}, \quad i, j, k, l=1 \ldots d_{\lambda} \text {. }
$$

Note, that for each fixed $i$ the elements $Y_{i j}^{\lambda} 1 \leq j \leq d_{\lambda}$ form a linear basis in the irreducible submodule $M_{\lambda}^{r}(i) \subset \bar{M}_{\lambda}$ generated by the primitive idempotent $Y_{i i}^{\lambda}$. And on the other hand, for each fixed $j$ the elements $Y_{i j}^{\lambda} 1 \leq i \leq d_{\lambda}$ form a linear basis in $M_{\lambda}^{l}(j)$ generated by $Y_{j j}^{\lambda}$. Therefore we have $\operatorname{dim} M_{\lambda}^{r}(i)=\operatorname{dim} M_{\lambda}^{l}(i)=d_{\lambda}$.

Otherwise stated, if we arrange the elements $Y_{i j}^{\lambda}$ into a rectangular $d_{\lambda} \times d_{\lambda}$ matrix, then its rows will represent the linear basises of $d_{\lambda}$ right submodules $M_{\lambda}^{r}(i)$, while the columns of the matrix will represent the linear basises of left submodules $M_{\lambda}^{l}(i)$. The diagonal entries of the matrix are the primitive idempotents $Y_{i i}^{\lambda}$.

An important property of $Y_{i i}^{\lambda}$ consists in the following fact. For any two idempotents $Y_{i i}^{\lambda}$ and $Y_{j j}^{\lambda}$ corresponding to the same partition $\lambda$ there exists an invertible element of $H_{m}$ which transforms one of these idempotents into the other one. This means that the submodules $M_{\lambda}^{r}(i)$ (or $M_{\lambda}^{l}(i)$ ) with different $i$ are isomorphic: they can be transformed into each other by left (respectively right) multiplication on some invertible element of Hecke algebra $H_{m}$. Note, that the whole $\bar{M}_{\lambda}$, being a two-sided submodule in $H_{m}$, is invariant with respect to such a multiplication.

In what follows we will not distinguish the isomorphic submodules corresponding to a partition $\lambda$ and will consider them up to an isomorphism. Then each submodule $M_{\lambda}^{r}(i) \subset \bar{M}_{\lambda}$ generated by $Y_{i i}^{\lambda}$ can be treated as the image of an irreducible $H_{m}$ module $M_{\lambda}^{r}$ (labelled only by the partition $\lambda \vdash m$ ) w.r.t. the following $H_{m}$ module monomorphism:

$$
M_{\lambda}^{r} \rightarrow M_{\lambda}^{r}(i) \subset \bar{M}_{\lambda} \subset H_{m}, \quad \lambda \vdash m .
$$

In this sense the submodule $\bar{M}_{\lambda}$ is isomorphic (as the $H_{m}$ module) to a direct sum of $d_{\lambda}$ copies of the module $M_{\lambda}^{r}$ :

$$
\bar{M}_{\lambda} \equiv \bigoplus_{i=1}^{d_{\lambda}} M_{\lambda}^{r}(i) \cong\left(M_{\lambda}^{r}\right)^{\oplus d_{\lambda}} .
$$

This formula reflects the well known fact that the regular representation of a finite dimensional semisimple algebra decomposes into a direct sum of irreducible modules and the multiplicity of each module is equal to its dimension.

\footnotetext{
${ }^{6}$ Matrix unit $E_{i j} 1 \leq i, j \leq m$ of the matrix algebra $\operatorname{Mat}_{m}(\mathbb{K})$ is the $m \times m$ matrix with the only nonzero entry at the intersection of the $i$-th row and the $j$-th column which is equal to 1 .
} 
Remark 1 It is worth mentioning that the module $M_{\lambda}^{r}$ can be mapped into $\bar{M}_{\lambda}$ in many different ways and submodules $M_{\lambda}^{r}(i)$ (generated by a fixed choice of primitive idempotents $\left\{Y_{i i}^{\lambda}\right\}$ ) are only particular cases of all possible monomorphisms. Indeed, let us take an arbitrary set of orthonormal $d_{\lambda} \times d_{\lambda}$ projectors $P^{(i)}$ of the rank 1 :

$$
P^{(i)} \cdot P^{(j)}=\delta^{i j} P^{(i)}, \quad \sum_{i=1}^{d_{\lambda}} P^{(i)}=\mathrm{id} .
$$

Then, as is evident from above relations and (2.9), the quantities $X_{i i}^{\lambda}=\sum_{r, s} P_{r s}^{(i)} Y_{r s}^{\lambda}$ are also a set of primitive idempotents in $H_{m}$ leading to another decomposition of $\bar{M}_{\lambda}$ into a direct sum of right submodules which will represent other possible monomorphisms of $M_{\lambda}^{r}$ into $\bar{M}_{\lambda}$.

Now with each right】 submodule $M_{\lambda}^{r}(i) \subset H_{m}$ we can associate a space $V_{\lambda}(i) \subset V^{\otimes m}$ in the following way:

$$
V_{\lambda}(i)=\operatorname{Im} \rho_{R}\left(M_{\lambda}^{r}(i)\right) .
$$

Besides, we will deal with a space $\bar{V}_{\lambda}$ which is the image of $\bar{M}_{\lambda}$ (2.8):

$$
\bar{V}_{\lambda}=\operatorname{Im} \rho_{R}\left(\bar{M}_{\lambda}\right) \subset V^{\otimes m} .
$$

The spaces $V_{\lambda}(i)$ with different $i$ (and all other $V_{\lambda}(X) \in \bar{V}_{\lambda}$ which are $\rho_{R}$-images of other possible monomorphisms of $M_{\lambda}^{r}$ into $\bar{M}_{\lambda}$ ) are isomorphic as vector spaces and we will not distinguish them. Instead, we will deal with a space $V_{\lambda}$ which (like $M_{\lambda}^{r}$ ) gives rise to a class of isomorphic embeddings $V_{\lambda} \hookrightarrow \bar{V}_{\lambda} \subset V^{\otimes m}$ and each $V_{\lambda}(i)$ (or any other $V_{\lambda}(X)$ as well) is just a particular representative of this class of isomorphic spaces.

Remark 2 Formula (2.11) has a nontrivial meaning for $m \geq 1$, whereas at $m=1$ the only space $V_{\lambda}$ is the space $V$ itself. For the future convenience we extend the formula to the case $k=0$. Namely, we will take by definition $V_{\lambda \vdash 0}=V_{0} \equiv \mathbb{K}$.

Now we can define the class of objects of the SW(V) category generated by a finite dimensional space $V$ over a field $\mathbb{K}$ equipped with a Hecke symmetry $R(\mathbb{1 . 6})-(1.8)$.

Definition 3 To each fixed nonnegative integer $k \in \mathbb{Z}_{+}$(for $k=0$ see remark g above) and each possible partition $\lambda \vdash k$ we put into correspondence a space $V_{\lambda}$ isomorphic to any $V_{\lambda}(i)$ in (2.11). The spaces $V_{\lambda}, \lambda \vdash k, k \in \mathbb{Z}_{+}$are the basic objects of the category $S W(V)$. The whole class of objects of the category is formed by direct sums of a finite number of basic objects $V_{\lambda}$. Thus, the spaces $V_{\lambda}(i)$ can be treated as the space $V_{\lambda}$ equipped with an embedding $\left\{V_{\lambda} \hookrightarrow \bar{V}_{\lambda} \subset V^{\otimes k}\right\}$.

Remark 4 The objects $V_{\lambda}$ should be simple objects of our category. However, some objects $V_{\lambda}$ of the category will be identified with each other (in particular, $V_{\left(1^{p}\right)}$ and $V_{0}$ ). So, finally simple objects of our category will be $V_{\lambda}$ modulo the mentioned identification. Up to this identification we will sometimes use the notation $\left[V_{\lambda}\right]_{k}$ for the family of embeddings $\left\{V_{\lambda} \hookrightarrow \bar{V}_{\lambda} \subset V^{\otimes k}\right\}$.

Let us turn now to the definition of morphisms of our category.

\subsection{Morphisms of the first kind and the structure of quasitensor category}

Let us denote $\operatorname{Mor}(U, V)$ the space of categorical morphisms $U \rightarrow V$.

\footnotetext{
${ }^{7}$ The choice of right submodules is made since we prefer to use the left action of $T \in \operatorname{End}(V)$ on $V$. Since an arbitrary element of $M_{\lambda}^{r}(i)$ has the form $Y_{i i}^{\lambda} f\left(\sigma_{1} \ldots \sigma_{m}\right)$ ( $f$ being a polynomial in the generators of $\left.H_{m}\right)$, then with such a choice the projector $\rho_{R}\left(Y_{i i}^{\lambda}\right)$ will be the last element acting on $V^{\otimes m}$ in formula (2.11).

${ }^{8}$ See Remark 1
} 
Definition 5 The morphisms of the first kind are defined as follows :

i) The set $\operatorname{Mor}\left(V_{\lambda}, V_{\lambda}\right)$ for any basic object $V_{\lambda}$ contains only multiples of the identical morphism. That is for any morphism $f: V_{\lambda} \rightarrow V_{\lambda}$ we have by definition $f=a$ id for some $a \in \mathbb{K}$.

ii)For an object embedded in $V^{\otimes k}$ the morphisms of the first kind are represented by a set of linear mappings:

$$
\forall k \in \mathbb{N}, \forall \tau \in H_{k}, \quad \phi^{k}(\tau)=\rho_{R}(\tau): \quad V^{\otimes k} \rightarrow V^{\otimes k} .
$$

Thus, being restricted to the set of subspaces $\left[V_{\lambda}\right]_{k}$ such a mapping $\phi^{k}$ sends each $V_{\lambda}(i) \in$ $\left[V_{\lambda}\right]_{k}$ to an isomorphic space or to zero spacef. This means that nontrivial mappings $\phi^{k}$ at most change the embedding of $V_{\lambda}$ into $V^{\otimes k}$ and therefore are multiples of identical morphism for the basic objects. Among the morphisms $\phi^{k}$ there is no one which would send $V_{\lambda}$ to $V_{\mu}$ with $\lambda \neq \mu$. A different kind of morphisms is considered in subsection 2.3.

Our next step consists in constructing a monoidal structure, which allows us to "multiply" the objects of the category. This means that we want to define a covariant functor

$$
\otimes: \mathrm{Ob}(\mathrm{SW}) \times \mathrm{Ob}(\mathrm{SW}) \rightarrow \mathrm{Ob}(\mathrm{SW})
$$

with some associativity morphisms (for detail see [CP, McL2]). As such a functor we take the usual tensor product of linear spaces with associativity morphisms to be identical. So, we have to prove that the tensor product of any two objects of our category is also an object, that is it can be decomposed into a direct sum of basic objects. Evidently, one only needs to verify this property for the tensor product of basic objects $V_{\lambda}$.

Proposition 6 For given $\lambda \vdash n$ and $\mu \vdash m$ the tensor product of two basic objects $V_{\lambda}$ and $V_{\mu}$ can be expanded into a direct sum of basic objects $V_{\nu}, \nu \vdash(n+m)$ :

$$
V_{\lambda} \otimes V_{\mu}=c_{\lambda \mu}^{\nu} V_{\nu}
$$

the coefficients $c_{\lambda \mu}^{\nu}$ being the Littlewood-Richardson ones entering the formula for product of Schur symmetric functions $s_{\lambda}$ in $p$ variablest $\mathrm{TP}$.

Proof. As is clear from the definition of basic objects $V_{\lambda}$ of our category the structure of their tensor product is controlled by that of modules $M_{\lambda}^{r}$ (2.10) and the assertion of the proposition can be reformulated in terms of these modules.

To do so let us consider an embedding of $V_{\lambda} \otimes V_{\mu}$ into $V^{\otimes(n+m)}$ of the form

$$
V_{\lambda} \otimes V_{\mu} \rightarrow V_{\lambda}(i) \otimes V_{\mu}(j) \subset V^{\otimes(n+m)}
$$

for some fixed $i$ and $j$. As was defined in (2.11) above, the space $V_{\lambda}(i)$ is the image of the right $H_{n}$ submodule $M_{\lambda}^{r}(i)$ under representation $\rho_{R}$ (2.1).

Consider an embedding $\mathcal{J}: H_{n} \times H_{m} \hookrightarrow H_{n+m}$ which on generators looks as follows:

$$
\begin{gathered}
\forall \sigma_{i}^{\prime} \in H_{n}: \mathcal{J}\left(\sigma_{i}^{\prime}\right)=\sigma_{i} \in H_{n+m} \quad 1 \leq i \leq n-1 \\
\forall \sigma_{j}^{\prime \prime} \in H_{m}: \quad \mathcal{J}\left(\sigma_{j}^{\prime \prime}\right)=\sigma_{n+j} \in H_{n+m}
\end{gathered}
$$

By construction $\mathcal{J}\left(H_{n}\right)$ and $\mathcal{J}\left(H_{m}\right)$ form two mutually commuting Hecke subalgebras in $H_{m+n}$.

Let $\lambda \vdash n, \mu \vdash m$ and $M_{\lambda}^{\prime}(i), M_{\mu}^{\prime \prime}(j)$ be two right submodules in $H_{n}$ and $H_{m}$ generated by the primitive idempotents $Y_{i i}^{\lambda}\left(\sigma^{\prime}\right)$ and $Y_{j j}^{\mu}\left(\sigma^{\prime \prime}\right)$ correspondingly. The image of the tensor product $M_{\lambda}^{\prime}(i) \otimes M_{\mu}^{\prime \prime}(j)$ under (2.14) is a right $H_{n} \times H_{m}$ module $M_{\lambda}^{r}(i) \otimes M_{\mu}^{r}(j) \subset H_{n+m}$ and obviously

$$
V_{\lambda}(i) \otimes V_{\mu}(j)=\operatorname{Im} \rho_{R}\left(M_{\lambda}^{r}(i) \otimes M_{\mu}^{r}(j)\right) .
$$

\footnotetext{
${ }^{9}$ For example, $\rho_{R}\left(\sigma_{i}+q^{-1} \operatorname{id}_{H}\right) 1 \leq i \leq k-1$ sends to zero the image of the antisymmetrizer $A^{(k)}$.

${ }^{10}$ The detailed description of Schur functions and related topics can be found in $\mathrm{M}$.
} 
The right $H_{n+m}$ module induced from $M_{\lambda}^{r}(i) \otimes M_{\mu}^{r}(j)$ is reducible and can be decomposed into a direct sum of irreducible right $H_{n+m}$ submodules $M_{\nu}^{r}(k)$. This decomposition is the image (under monomorphism $M_{\lambda}^{r} \rightarrow M_{\lambda}^{r}(i)$ ) of the following relation between irreducible modules $M_{\lambda}^{r}$ and $M_{\mu}^{r}$ :

$$
M_{\lambda}^{r} \otimes M_{\mu}^{r}=c_{\lambda \mu}^{\nu} M_{\nu}^{r}
$$

where the coefficients $c_{\lambda \mu}^{\nu}$ are equal to the multiplicity of irreducible $H_{n+m}$ characters $\chi^{\nu}$ in the character induced from $\chi^{\lambda} \times \chi^{\mu}$. Due to (2.15) the coefficients $c_{\lambda \mu}^{\nu}$ will also determine the expansion of tensor product of two basic objects $V_{\lambda}$ and $V_{\mu}$.

Now we use the fact that at generic $q$ the Hecke algebra $H_{m}$ is isomorphic to the group algebra $\mathbb{K}\left(S_{m}\right)$ of the $m$-th order permutation group $S_{m}$ for all $m \geq 1$. Therefore, the coefficients $c_{\lambda \mu}^{\nu}$ defining the multiplicity of irreducible module $M_{\nu}^{r}$ in the tensor product $M_{\lambda}^{r} \otimes M_{\mu}^{r}$ are the same for the Hecke algebra and for the algebra $\mathbb{K}\left(S_{m}\right)$. To complete the proof, note that as is well known from the representation theory of symmetric group (see for example [M]) the corresponding multiplicities coincide with the Littlewood-Richardson coefficients in the product of symmetric Schur functions $s_{\lambda}$.

Remark 7 The explicit calculation of the decomposition of $M_{\lambda}^{r}(i) \otimes M_{\mu}^{r}(j)$ into a direct sum of $H_{n+m}$ submodules gives

$$
M_{\lambda}^{r}(i) \otimes M_{\mu}^{r}(j)=\alpha_{\lambda \mu}^{\nu}(k(i, j)) M_{\nu}^{r}(k(i, j)), \quad \alpha_{\lambda \mu}^{\nu}(k(i, j)) \in \mathbb{Z}_{+} .
$$

But the isomorphic submodules $M_{\nu}^{r}(k)$ with different $k$ are images of the same module $M_{\nu}^{r}$ equipped with different monomorphisms (depending on $i$ and $j$ ) $M_{\nu}^{r} \rightarrow \bar{M}_{\nu} \subset H_{n+m}$. The sum $\sum_{k} \alpha_{\lambda \mu}^{\nu}(k)$ does not depend on $i$ and $j$ and is equal to $c_{\lambda \mu}^{\nu}$ which defines the structure of the tensor product (2.16).

Now we should convert the monoidal category SW(V) into a quasitensor one (see $\mathrm{CP}]$ ). This means, that we need to define a set of natural commutativity (or braiding) isomorphisms (1.2) for any pair $U, V \in \mathrm{Ob}(\mathrm{SW}(\mathrm{V}))$. To be compatible with the monoidal structure the braiding isomorphisms must satisfy the following property for any triple $U, V, W \in \mathrm{Ob}(\mathrm{SW}(\mathrm{V}))$ :

$$
R_{U, V \otimes W}=\left(\operatorname{id}_{V} \otimes R_{U, W}\right) \circ\left(R_{U, V} \otimes \mathrm{id}_{W}\right) \quad R_{V \otimes W, U}=\left(R_{V, U} \otimes \mathrm{id}_{W}\right) \circ\left(\operatorname{id}_{V} \otimes R_{W, U}\right) .
$$

Let us recall that the associativity isomorphisms are taken to be identical.

Besides, considering two possible ways of transformation of $U \otimes V \otimes W$ into $W \otimes V \otimes U$ one comes to the following condition:

$$
\left(R_{V, W} \otimes \mathrm{id}_{U}\right) \circ\left(\mathrm{id}_{V} \otimes R_{U, W}\right) \circ\left(R_{U, V} \otimes \mathrm{id}_{W}\right)=\left(\mathrm{id}_{W} \otimes R_{U, V}\right) \circ\left(R_{U, W} \otimes \mathrm{id}_{V}\right) \circ\left(\mathrm{id}_{U} \otimes R_{V, W}\right) .
$$

Let us begin with the simplest object - the space $V$. As a braiding isomorphism for $V \otimes V$ we take the Hecke symmetry $R$. Requirement (2.18) on $V^{\otimes 3}$ transforms into the Yang-Baxter equation (1.7) on $R$ which is satisfied by the definition of $R$. The decomposition property (2.17) will be used as a definition of braiding isomorphisms for an arbitrary tensor power of the space $V$. That is we take:

$$
R_{V, V \otimes k}=R_{k} R_{k-1} \ldots R_{1} \quad R_{V^{\otimes k}, V}=R_{1} R_{2} \ldots R_{k} .
$$

In the above formula a shorthand notation $R_{i} \equiv R_{i i+1}$ is used. Introducing one more notation for the chain of $R$ matrices

$$
R_{i \rightarrow j} \equiv \begin{cases}R_{i} R_{i+1} \ldots R_{j} & \text { if } j=i+n \geq i \\ R_{i} R_{i-1} \ldots R_{j} & \text { if } j=i-n \leq i\end{cases}
$$


we can write a compact form for the braiding isomorphism in the general case:

$$
\begin{aligned}
R_{V^{\otimes n}, V \otimes m} & =R_{m \rightarrow(m+n-1)} R_{(m-1) \rightarrow(m+n-2)} \ldots R_{1 \rightarrow n} \\
& \equiv R_{m \rightarrow 1} R_{(m+1) \rightarrow 2} \ldots R_{(n+m-1) \rightarrow n} .
\end{aligned}
$$

The two parts of this formula correspond to two possible ways of passing from $V^{\otimes n} \otimes V^{\otimes m}$ to $V^{\otimes m} \otimes V^{\otimes n}$. Note, that all mappings (2.20) are morphisms of the first kind.

As for the braiding isomorphisms for $\mathbb{K} \otimes V$ we take it to be the usual flip:

$$
\mathbb{K} \otimes V=V \otimes \mathbb{K}=V .
$$

By definition of the objects of the category SW(V) any tensor power $V^{\otimes m}$ can be decomposed into a direct sum of basic objects $V_{\lambda}, \lambda \vdash m$. Therefore by making use of formula (2.20) one can define the braiding isomorphism for the tensor product of two arbitrary basic objects $V_{\lambda} \otimes V_{\mu}$, (and, therefore, for the tensor product of any couple of objects) if we manage to prove that isomorphism (2.20) does not "destroy" the structure of embeddings of $V_{\lambda} \otimes V_{\mu}$ into $V^{\otimes n} \otimes V^{\otimes m}$. If it is the case then we can take the restriction of (2.20) onto $\bar{V}_{\lambda} \otimes \bar{V}_{\mu}$ as the braiding morphism for the tensor product of $V_{\lambda}$ and $V_{\mu}$.

That is we have to show the following. Consider two arbitrary embeddings $\mathcal{J}_{i}: V_{\lambda} \hookrightarrow V^{\otimes n}$ and $\mathcal{J}_{j}: V_{\mu} \hookrightarrow V^{\otimes m}$. Let $\mathcal{J}_{i}\left(V_{\lambda}\right)=V_{\lambda}(i)$ and $\mathcal{J}_{j}\left(V_{\mu}\right)=V_{\mu}(j)$. Let us recall that $V_{\lambda}(i)$ is defined by (2.11). We want to show that under isomorphism (2.20) one gets:

$$
V_{\lambda}(i) \otimes V_{\mu}(j) \longrightarrow R_{\lambda \mu}\left(V_{\lambda}(i) \otimes V_{\mu}(j)\right)
$$

where $R_{\lambda \mu}$ is an invertible operator from $\operatorname{End}\left(\bar{V}_{\lambda} \otimes \bar{V}_{\mu}\right)$ (the space $\bar{V}_{\lambda}$ is defined in (2.12)) which does not depend on $i$ and $j$.

Proposition 8 For two given partitions $\lambda \vdash n, \mu \vdash m$ and two arbitrary integers $1 \leq i \leq d_{\lambda}$ and $1 \leq j \leq d_{\mu}$ consider the corresponding spaces $V_{\lambda}(i)$ and $V_{\mu}(j)$ as defined in (2.11). Then under isomorphism (2.20) one has

$$
V_{\lambda}(i) \otimes V_{\mu}(j) \longrightarrow R_{\lambda \mu}\left(V_{\lambda}(i) \otimes V_{\mu}(j)\right)
$$

where the operator $R_{\lambda \mu}$ is defined on $\bar{V}_{\lambda} \otimes \bar{V}_{\mu}$ by means of the following formula:

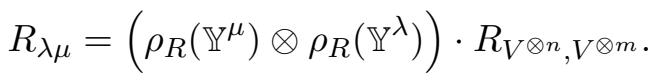

Here $R_{V \otimes n, V \otimes m}$ is defined by (2.2Q) and

$$
\mathbb{Y}^{\lambda} \equiv \sum_{i=1}^{d_{\lambda}} Y_{i i}^{\lambda}
$$

is a central idempotent of the corresponding Hecke algebra.

Proof. The proof is based on the embedding of $H_{n} \times H_{m}$ into $H_{n+m}$ introduced in Proposition 6. In accordance with (2.14) and (2.15) we can write

$$
V_{\lambda}(i) \otimes V_{\mu}(j)=\operatorname{Im}\left\{\rho_{R}\left(Y_{i i}^{\lambda}\right) \otimes \rho_{R}\left(Y_{j j}^{\mu}\right)\right\} .
$$

Considered on the arbitrary basis element of the space $V^{\otimes n} \otimes V^{\otimes m}$ the right hand side of the above formula reads:

$$
e_{\langle 1|} \otimes \ldots \otimes e_{\langle n+m|} \rightarrow e_{\langle 1|} \otimes \ldots \otimes e_{\langle n+m|}\left\{\mathcal{Y}_{i i}^{\lambda} \otimes \mathcal{Y}_{j j}^{\mu}\right\}_{\langle 1 \ldots n+m|}^{|1 \ldots n+m\rangle}
$$


where the matrices $\mathcal{Y}_{i i}^{\lambda}=\rho_{R}\left(Y_{i i}^{\lambda}\right)$ and $\mathcal{Y}_{j j}^{\mu}=\rho_{R}\left(Y_{j j}^{\mu}\right)$ are some polynomials in $R_{1} \ldots R_{n-1}$ and $R_{n+1} \ldots R_{n+m-1}$ respectively:

$$
\mathcal{Y}_{i i}^{\lambda}=\mathcal{Y}_{i i}^{\lambda}\left(R_{1}, \ldots, R_{n-1}\right), \quad \mathcal{Y}_{j j}^{\mu}=\mathcal{Y}_{j j}^{\mu}\left(R_{n+1}, \ldots, R_{n+m-1}\right) .
$$

Applying the braiding isomorphism (2.20) to $V^{\otimes n} \otimes V^{\otimes m}$ leads to the following result for formula (2.24) :

$$
e_{\langle 1|} \otimes \ldots \otimes e_{\langle n+m|} \rightarrow e_{\langle 1|} \otimes \ldots \otimes e_{\langle n+m|}\left\{R_{V \otimes n, V \otimes m} \cdot\left(\mathcal{Y}_{i i}^{\lambda} \otimes \mathcal{Y}_{j j}^{\mu}\right)\right\}
$$

where the symbol $\cdot$ stands for the matrix multiplication.

Now one should take into account the following relations which are direct consequence of (2.20) and Yang-Baxter equation (1.7):

$$
\begin{array}{ll}
R_{V^{\otimes n}, V \otimes m} \cdot R_{i}=R_{i+m} \cdot R_{V^{\otimes n}, V \otimes m} & 1 \leq i \leq n-1 \\
R_{V^{\otimes n}, V \otimes m} \cdot R_{j}=R_{j-n} \cdot R_{V^{\otimes n}, V \otimes m} & n+1 \leq j \leq n+m-1 .
\end{array}
$$

Using these relations one gets:

$$
\begin{aligned}
& R_{V \otimes n, V \otimes m} \cdot \mathcal{Y}_{i i}^{\lambda}\left(R_{1} \ldots R_{n-1}\right) \otimes \mathcal{Y}_{j j}^{\mu}\left(R_{n+1} \ldots R_{n+m-1}\right)= \\
& \mathcal{Y}_{j j}^{\mu}\left(R_{1} \ldots R_{m-1}\right) \otimes \mathcal{Y}_{i i}^{\lambda}\left(R_{m+1} \ldots R_{m+n-1}\right) \cdot R_{V \otimes n}, V^{\otimes m} .
\end{aligned}
$$

This formula proves (2.22). In order to find the form of $R_{\lambda \mu}$ we observe that $\mathbb{Y}^{\lambda}=\sum_{i} Y_{i i}^{\lambda}$ are central elements of $H_{n}(\lambda \vdash n)$ and besides $Y_{i i}^{\lambda} \equiv Y_{i i}^{\lambda} \mathbb{Y}^{\lambda} \forall i, j$. Therefore

$$
\left(\mathcal{Y}_{j j}^{\mu} \otimes \mathcal{Y}_{i i}^{\lambda}\right) \cdot R_{V^{\otimes n}, V \otimes m} \equiv \mathcal{Y}_{j j}^{\mu} \otimes \mathcal{Y}_{i i}^{\lambda} \cdot\left(\rho_{R}\left(\mathbb{Y}^{\mu}\right) \otimes \rho_{R}\left(\mathbb{Y}^{\lambda}\right) \cdot R_{V^{\otimes n}, V \otimes m}\right) .
$$

where $\rho_{R}\left(\mathbb{Y}^{\lambda}\right)$ is the projector onto $\bar{V}_{\lambda} \subset V^{\otimes(n+m)}$. Thus, we come to form (2.23) of $R_{\lambda \mu}$.

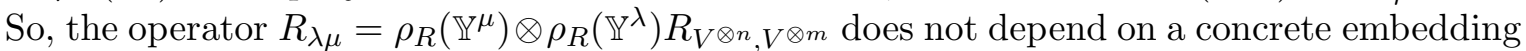
of $V_{\lambda} \otimes V_{\mu} \in \mathrm{Ob}(\mathrm{SW}(\mathrm{V}))$ into $V^{n} \otimes V^{m}$ (i.e., it does not depend on indices $i, j$ ) and represents a braiding isomorphism for $V_{\lambda} \otimes V_{\mu}$.

\section{3 $\quad$ Morphisms of the second kind and reduction procedure}

As was mentioned above (see (2.6)) the image of the antisymmetrizer $A^{m}$ with $m>p$ is identical zero in $V^{\otimes m}$. It can be shown that the same is true for any $\operatorname{Im} \rho_{R}\left(M_{\lambda}^{r}\right)$ in case if the height $\ell(\lambda)>p$. This means that the basic objects of our category $V_{\lambda}$ are labelled by partitions with restricted height: $V_{\lambda} \not \equiv 0 \Leftrightarrow \ell(\lambda) \leq p$.

However, there is another consequence of (2.5) - (2.6) which concerns the objects labelled by partitions with the height equal to $p$. Consider a partition $\lambda \vdash(p+m)$ for some nonnegative integer $m$ such that $\ell(\lambda)=p$. Let $\mu$ denote a partition of $m$ which is obtained from $\lambda$ by striking the first column out of the Young diagram corresponding to $\lambda$. The parts of $\lambda$ and $\mu$ are connected by the relation $\mu_{i}=\lambda_{i}-1, \forall \lambda_{i} \neq 0$. As immediately follows from Proposition 6 and from the above remark about the maximal height of $\lambda$ the following decomposition takes place:

$$
V_{\lambda} \cong V_{\left(1^{p}\right)} \otimes V_{\mu}
$$

where $V_{\left(1^{p}\right)}$ stands for the one dimensional space labelled by one-column diagram with $p$ boxes: $\lambda=\left(1^{p}\right)$.

Isomorphisms (2.25) allow us to define the so called reduction procedure and to introduce morphisms of the second kind in our category, namely, those identifying $V_{\lambda}$ and $V_{\mu}$. For this purpose consider a mapping $\psi$ sending the one-dimensional space $V_{\left(1^{p}\right)}$ into the field $\mathbb{K}$. Evidently, 
one only needs to fix the action of the mapping $\psi$ on a basis vector of $V_{\left(1^{p}\right)} \cong \operatorname{Im} \rho_{R}\left(A^{(p)}\right)$. In the general form such a mapping looks like

$$
\psi\left(e_{\langle 1|} \otimes \ldots \otimes e_{\langle p|} v^{|1 \ldots p\rangle}\right)=1 \in \mathbb{K} .
$$

The choice of 1 in the above formula does not restrict generality of our consideration.

Let us suppose that $\psi \in \operatorname{Mor}\left(V_{\left(1^{p}\right)}, \mathbb{K}\right)$. Then taking into account $(1.3)$ and the fact that the identity operator is a categorical morphism we conclude that the following diagrams must be commutative:

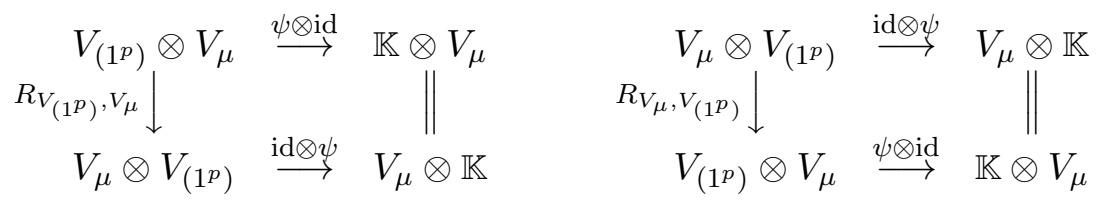

Consider a particular case of these diagrams by putting $V_{\mu}=V$ (if they are commutative in this case the same will be true for any $\mu$ ). Then by passing to the basis we have

$$
V_{\left(1^{p}\right)} \otimes V: \quad e_{\langle 1|} \otimes \ldots \otimes e_{\langle p|} \otimes e_{\langle p+1|} v^{|1 \ldots p\rangle} \stackrel{\psi \otimes \mathrm{id}}{\longrightarrow} 1 \otimes e_{\langle p+1|}=e_{\langle p+1|} \otimes 1 .
$$

According to another way in the left diagram we should first apply the braiding morphism $R_{V_{\left(1^{p}\right)}, V}$. Thus, we have

$$
\begin{aligned}
R_{V_{(1 p)}, V}: \quad e_{\langle 1|} \otimes \ldots \otimes e_{\langle p|} \otimes e_{\langle p+1|} v^{|1 \ldots p\rangle} & \longrightarrow \\
& e_{\langle 1|} \otimes \ldots \otimes e_{\langle p|} \otimes e_{\langle p+1|} R_{1} \ldots R_{p} v^{|1 \ldots p\rangle} .
\end{aligned}
$$

In order to simplify this expression we need the following useful relations (see [HIOPT]):

$$
\begin{aligned}
& R_{1} \ldots R_{p} \mathcal{A}^{(p)}=(-1)^{p-1} q p_{q} \mathcal{A}^{(2, p+1)} \mathcal{A}^{(p)} \\
& R_{p} \ldots R_{1} \mathcal{A}^{(2, p+1)}=(-1)^{p-1} q p_{q} \mathcal{A}^{(p)} \mathcal{A}^{(2, p+1)} \\
& \mathcal{A}^{(p)} \mathcal{A}^{(2, p+1)} \mathcal{A}^{(p)}=p_{q}^{-2} \mathcal{A}^{(p)} \otimes \mathrm{id}_{p+1} .
\end{aligned}
$$

In these formulas the antisymmetrizer $\mathcal{A}^{(2, p+1)}$ has the same form as $\mathcal{A}^{(p)}$ but it depends on $R_{2}, \ldots, R_{p}$ instead of $R_{1}, \ldots, R_{p-1}$.

Now in virtue of (2.28) we obtain:

$$
R_{1} \ldots R_{p} v^{|1 \ldots p\rangle}=v^{|2 \ldots p+1\rangle} N_{\langle p+1|}^{|1\rangle}
$$

where the matrix $N$ is defined as follows:

$$
N_{j}^{i} \equiv(-1)^{p-1} q p_{q} u_{a_{2} \ldots a_{p} j} v^{i a_{2} \ldots a_{p}} \quad \text { or } \quad N_{\left\langle 1^{\prime}\right|}^{|1\rangle}=(-1)^{p-1} q p_{q} u_{\left\langle 2 \ldots p 1^{\prime}\right|} v^{|12 \ldots p\rangle} .
$$

Therefore the composition (id $\otimes \psi) \circ R_{V_{\left(1^{p}\right)}, V}$ gives the following result:

$$
e_{\langle 1|} \otimes \ldots \otimes e_{\langle p|} \otimes e_{\langle p+1|} v^{|1 \ldots p\rangle} \rightarrow e_{\langle 1|} \otimes \ldots \otimes e_{\langle p|} \otimes e_{\langle p+1|} v^{|2 \ldots p+1\rangle} N_{\langle p+1|}^{|1\rangle} \rightarrow e_{\langle 1|} N_{\langle p+1|}^{|1\rangle} \otimes 1
$$

which obviously differs from (2.27). These results can be made compatible iff $N$ is a scalar matrix (i.e., it is proportional to the $n \times n$ unit matrix $I$ ). If it is so, then by multiplying the braiding $R$ by an appropriate factor we can achieve the commutativity of the left diagram above. Indeed, if we replace the braiding $R$ by $a R, a \in \mathbb{K}$, the factor $a^{p}$ appears in the right hand side of (2.29). Choosing properly the factor $a$ we can obtain $a^{p} N=I$. 
Applying the same procedure for the space $V \otimes V_{\left(1^{p}\right)}$ (the right diagram above) we find:

$$
e_{\langle 1|} \otimes e_{\langle 2|} \otimes \ldots \otimes e_{\langle p+1|} v^{|2 \ldots p+1\rangle} \longrightarrow 1 \otimes e_{\langle p+1|} M_{\langle 1|}^{|p+1\rangle}
$$

where

$$
M_{j}^{i} \equiv(-1)^{p-1} q p_{q} u_{j a_{2} \ldots a_{p}} v^{a_{2} \ldots a_{p} i} \quad \text { or } \quad M_{\langle 1|}^{\left|1^{\prime}\right\rangle} \equiv(-1)^{p-1} q p_{q} u_{\langle 12 \ldots p|} v^{\left|2 \ldots p 1^{\prime}\right\rangle} \text {. }
$$

For the same reason we assume the matrix $M$ to be scalar. Then the right diagram can also be made commutative by a proper rescaling of $R$. However, if we want to obtain the unit matrix instead of the matrices $N$ and $M$ simultaneously we have to impose one more condition: $M=N$.

Therefore from now on we will suppose the Hecke symmetry $R$ to satisfy the relation

$$
M=N=a I, \quad a \in \mathbb{K}^{\times} .
$$

Thus, the mapping $\psi$ becomes a morphism of the category SW(V) after a proper renormalization of the braiding morphisms.

This normalization factor is easy to find. Indeed, using relations (2.28) (valid for any Hecke symmetry of the rank $p$ ) we find

$$
M \cdot N=q^{2} I
$$

So, if relation (2.31) is satisfied then by virtue of (2.32) we have $a= \pm q$ and hence

$$
N=M= \pm q I
$$

Therefore by assuming $\psi$ to be a morphism and the braiding to be natural we should pass from the initial Hecke symmetry $R$ to the braiding

$$
\bar{R}=( \pm q)^{-\frac{1}{p}} R \text {. }
$$

On the higher tensor powers this renormalization gives rise to a renormalized braiding as well

$$
\bar{R}_{V^{\otimes n}, V \otimes m}=( \pm q)^{-\frac{m n}{p}} R_{V^{\otimes n}, V \otimes m} .
$$

The mapping $\psi$ (as well as its inverse $\psi^{-1}$ ) will be called a morphism of the second kind.

Now let us compare our approach to introducing categorical morphisms with that making use of the RTT algebra. Recall that this algebra denoted $\mathcal{T}$ is generated by $n^{2}$ elements $T_{j}^{i}$ subject to the relations [FRT:

$$
R_{12} T_{1} T_{2}=T_{1} T_{2} R_{12}
$$

It becomes a bialgebra being equipped with a coproduct $\Delta$ and a counit $\varepsilon$ as follows:

$$
\Delta\left(T_{j}^{i}\right)=T_{k}^{i} \otimes T_{j}^{k} \quad \varepsilon\left(T_{j}^{i}\right)=\delta_{j}^{i} .
$$

Define the right comodule structure $\delta_{r}: V \rightarrow V \otimes \mathcal{T}$ on the space $V$ as follows:

$$
\delta_{r}(v)=e_{k} \otimes T_{i}^{k} v^{i}, \quad \forall v=v^{i} e_{i} \in V .
$$

Such a coaction is extended to $V^{\otimes k}$ in the obvious way:

$$
\delta_{r}: V^{\otimes k} \rightarrow V^{\otimes k} \otimes \mathcal{T} \quad e_{\langle 1|} \otimes \ldots \otimes e_{\langle k|} \rightarrow e_{\langle 1|} \otimes \ldots \otimes e_{\langle k|} \otimes T_{1} \ldots T_{k}
$$

\footnotetext{
${ }^{11}$ Note that the matrices $N$ and $M$ differ by a factor from those considered in $\mathrm{G}$.
} 
and all the comodule properties are easily verified. Then each $V_{\lambda}(i) \lambda \vdash k$ turns out to be an invariant subcomodule in $V^{\otimes k}$ since the coaction $\delta_{r}$ commute with the action of $\rho_{R}\left(M_{\lambda}^{r}(i)\right)$ on $V^{\otimes k}$ :

$$
\forall k, \forall \lambda \vdash k, \quad \rho_{R}\left(M_{\lambda}^{r}\right) T_{1} T_{1} \ldots T_{k}=T_{1} T_{1} \ldots T_{k} \rho_{R}\left(M_{\lambda}^{r}\right) .
$$

This equation is a direct consequence of defining relations (2.36) and explicit formula for primitive idempotents of $H_{k}$ which expresses each $Y_{i i}^{\lambda}$ as a polynomial in $\sigma_{1}, \sigma_{2}, \ldots, \sigma_{k-1}$.

Thus, any object of the category $\mathrm{SW}(\mathrm{V})$ can be equipped with a $\mathcal{T}$-coaction. This structure is often used in order to define categorical morphisms: one says that a map $U \rightarrow V$ is a morphism if it commutes with this coaction. Let us analyze what it entails being applied to $\psi$. For this purpose consider a quantum determinant $\operatorname{det}_{q} T$ of bialgebra (2.36) defined as follows FRT:

$$
\operatorname{det}_{q} T \equiv u_{\langle 12 \ldots p|} T_{1} T_{2} \ldots T_{p} v^{|12 \ldots p\rangle} .
$$

The commutation relations of $\operatorname{det}_{q} T$ with generators of (2.36) read (cf. G ):

$$
\operatorname{det}_{q} T \cdot T=\left(N^{-1} T N\right) \cdot \operatorname{det}_{q} T \Leftrightarrow \operatorname{det}_{q} T \cdot(N T)=(T N) \cdot \operatorname{det}_{q} T
$$

or due to $(2.32)$

$$
\operatorname{det}_{q} T \cdot T=\left(M T M^{-1}\right) \cdot \operatorname{det}_{q} T \Leftrightarrow \operatorname{det}_{q} T \cdot(T M)=(M T) \cdot \operatorname{det}_{q} T .
$$

If we want the mapping $\psi(2.26)$ to commute with the comodule structure we have to introduce one more condition:

$$
\operatorname{det}_{q} T=1 \text {. }
$$

But such a relation is compatible with the algebraic structure only iff $\operatorname{det}_{q} T$ is a central element of algebra (2.36). By virtue of (2.39) this means that the matrix $N$ (and hence $M$ ) must be scalar.

Formally, the condition " $N$ and $M$ are scalar" is weaker than " $N$ and $M$ are scalar and equal to each other". It is not clear whether there exist Hecke symmetries such that the matrices $N$ and $M$ are scalar but not equal to each other. Nevertheless, the family of even Hecke symmetries satisfying (2.33) is sufficiently large. It will be shown by one of the authors (R.L) in a separate paper.

Let us also remark that for the quasiclassical Hecke symmetries coming from the universal $R$ matrix of QG $U_{q}(s l(n))$ condition (2.31) holds true. The quantum determinant $\operatorname{det}_{q} T$ and the unit element 1 generate the center of (2.36) [FRT. But in general (even in the quasiclassical case) $\operatorname{det}_{q} T$ is not central.

\subsection{The structure of rigid category}

To convert a (quasi)tensor category $\mathcal{C}$ into a rigid one we must specify the following data $\overline{\mathrm{CP}}$ :

a. A mapping $*: \mathrm{Ob}(\mathcal{C}) \rightarrow \mathrm{Ob}(\mathcal{C})$ which for any $U \in \mathrm{Ob}(\mathcal{C})$ put into correspondence its left dual $U^{*}$

b. For any pair of dual objects $U$ and $U^{*}$ there exist evaluation $\mathbf{e v}_{U}$ and coevaluation $\boldsymbol{\pi}_{U}$ morphisms of $\mathcal{C}$

$$
\boldsymbol{\pi}_{U}: \mathbb{K} \longrightarrow U \otimes U^{*}, \quad \mathbf{e v}_{U}: U^{*} \otimes U \longrightarrow \mathbb{K}
$$

such that the following diagrams are commutative:
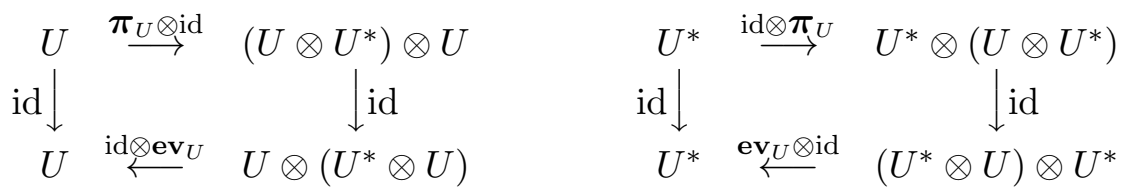
Let us begin with the case $U=V$. By our construction of the morphism $\psi$ it is evident that the dual space $V^{*}$ is nothing but $\Lambda^{p-1}(V)$. So, in order to satisfy (2.41) we should only fix a convenient basis converting $\Lambda^{p-1}(V)$ into a left dual space.

Definition 9 The space $\Lambda^{p-1}(V)$ equipped with the basis

$$
e^{i}=e_{a_{2}} \otimes \ldots \otimes e_{a_{p}} v^{i a_{2} \ldots a_{p}} \quad \text { or } \quad e^{|1\rangle}=e_{\langle 2|} \otimes \ldots \otimes e_{\langle p|} v^{|12 \ldots p\rangle} .
$$

will be called a left dual space to the space $V$. The morphisms $\mathbf{e v}_{V}$ and $\boldsymbol{\pi}_{V}$ are defined as follows (on basis vectors):

$$
\begin{array}{ll}
\mathbf{e v}_{V}: & e^{i} \otimes e_{j} \rightarrow \delta_{i}^{i} 1 \\
\boldsymbol{\pi}_{V}: & 1 \longrightarrow e_{i} \otimes e^{i} .
\end{array}
$$

In order to justify this definition we have to show the following.

Proposition 10 Mappings (2.43) are morphisms of the $S W(V)$ category and they satisfy property (2.41).

Proof. The fact that $\boldsymbol{\pi}_{V}$ is a morphism is evident since

$$
\boldsymbol{\pi}_{V}=\psi^{-1} .
$$

To show that $\mathbf{e v}_{V}$ is a morphism let us consider the mapping

$$
\varphi=(-1)^{p-1} p_{q} \psi \circ \mathcal{A}^{p}: \quad V^{\otimes p} \rightarrow \mathbb{K} .
$$

It is a morphism by construction. In a basis form we get

$$
\varphi: e_{i_{1}} \otimes \ldots \otimes e_{i_{p}} \rightarrow(-1)^{p-1} p_{q} u_{i_{1} \ldots i_{p}} .
$$

Applying this morphism to the element

$$
e^{i} \otimes e_{j}=v^{i a_{2} \ldots a_{p}} e_{a_{2}} \otimes \ldots \otimes e_{a_{p}} \otimes e_{j}
$$

we get

$$
\varphi\left(e^{i} \otimes e_{i}\right)=(-1)^{p-1} p_{q} v^{i a_{2} \ldots a_{p}} u_{a_{2} \ldots a_{p} j}=q^{-1} N_{j}^{i}= \pm \delta_{j}^{i} .
$$

Thus, the mapping $\mathbf{e v}_{V}$ is nothing but the morphism $\pm \varphi$ restricted on $\Lambda^{p-1}(V) \otimes V$.

Diagrams (2.41) are obviously commutative.

Let us define now left duals to other simple objects of our category. First, introduce a dual object to $V^{\otimes m}$ by putting $\left(V^{\otimes m}\right)^{*}=\left(V^{*}\right)^{\otimes m}$.

Proposition 11 The space $\left(V^{*}\right)^{\otimes m}$ is dual to the space $\left(V^{\otimes m}\right)$ being equipped with the mappings

$$
\mathbf{e v}_{U}: V^{* \otimes m} \otimes V^{\otimes m} \longrightarrow \mathbb{K}, \quad \boldsymbol{\pi}_{U}: \mathbb{K} \longrightarrow V^{\otimes m} \otimes V^{* \otimes m}
$$

which are defined to be:

$$
\begin{array}{ll}
\mathbf{e v}_{U}: & e^{|m\rangle} \otimes \ldots \otimes e^{|1\rangle} \otimes e_{\langle 1|} \otimes \ldots e_{\langle m|} \rightarrow \delta_{\langle 1|}^{|1\rangle} \ldots \delta_{\langle m|}^{|m\rangle} . \\
\boldsymbol{\pi}_{U}: & 1_{\mathbb{K}} \rightarrow e_{\langle 1|} \otimes \ldots \otimes e_{\langle m|} \otimes e^{|m\rangle} \otimes \ldots \otimes e^{|1\rangle}
\end{array} .
$$

Proof is obvious.

Now we are ready to define the dual $V_{\lambda}^{*}$ and corresponding morphisms $\boldsymbol{\pi}_{V_{\lambda}}$ and $\mathbf{e v}_{V_{\lambda}}$ for arbitrary basic object $V_{\lambda}$ of the category $\mathrm{SW}(\mathrm{V})$. 
Fix a basic object $V_{\lambda}, \lambda \vdash m$, and consider its particular embedding into $V^{\otimes m}$ in the form (2.11):

$$
V_{\lambda}(i)=\operatorname{Im} \rho_{R}\left(Y_{i i}^{\lambda}\right)
$$

This space is spanned by the following vectors

$$
e_{\langle 1|} \otimes \ldots \otimes e_{\langle m|} \cdot\left(\mathcal{Y}_{i i}^{\lambda}\right)^{|1 \ldots m\rangle}\langle 1 \ldots m|
$$

where $\mathcal{Y}_{i i}^{\lambda}$ is a matrix of $Y_{i i}^{\lambda}$ in the representation $\rho_{R}$.

Let us define a space $V_{\lambda}^{*}(i) \subset V^{* \otimes m}$ as a linear span of the form:

$$
V_{\lambda}^{*}(i)=\left\{w^{*}: w^{*}=w_{\langle 1 \ldots m|}\left(\mathcal{Y}_{i i}^{\lambda}\right) \cdot e^{|m\rangle} \otimes \ldots \otimes e^{|1\rangle}\right\}
$$

where $w_{\langle 1 \ldots m|}$ is an arbitrary tensor with components from $\mathbb{K}$. It is important, that the order of spaces in this formula is reversed comparing with the preceding relation while the matrix $\mathcal{Y}_{i i}^{\lambda}$ is the same.

Finally, we have the following.

Proposition 12 The space $V_{\lambda}^{*}$ being equipped with the morphisms $\mathbf{e v}_{V_{\lambda}}$ and $\boldsymbol{\pi}_{V_{\lambda}}$ of the form

$$
\begin{array}{ll}
\mathbf{e v}_{V_{\lambda}}: & V_{\lambda}^{*} \otimes V_{\lambda} \longrightarrow \mathcal{Y}_{i i}^{\lambda} \\
\boldsymbol{\pi}_{V_{\lambda}}: & 1_{\mathbb{K}} \longrightarrow e_{\langle 1|} \otimes \ldots e_{\langle m|} \otimes \mathcal{Y}_{i i}^{\lambda} \cdot e^{|m\rangle} \otimes \ldots \otimes e^{|1\rangle} .
\end{array}
$$

becomes left dual to the space $V_{\lambda}$ (provided that $V_{\lambda}$ is realized as $V_{\lambda}(i)$ ).

Let us note that in fact the space $V_{\lambda}^{*}$ is nothing but

$$
V_{\mu}, \quad \mu=\left(\lambda_{1}, \lambda_{1}-\lambda_{p-1}, \lambda_{1}-\lambda_{p-2}, \ldots, \lambda_{1}-\lambda_{2}\right)
$$

equipped with an embedding in the space $V^{* \otimes m}$. This shows that we do not need to construct left dual to the space $V_{\lambda}^{*}$ because it is just the object $V_{\lambda}$.

Nevertheless, the problem of an explicit pairing

$$
V_{\lambda} \otimes V_{\lambda}^{*} \rightarrow \mathbb{K}
$$

converting $V_{\lambda}^{*}$ into the right dual to $V_{\lambda}$ (and $V_{\lambda}$ in the left dual to $V_{\lambda}^{*}$ ) is very important. In order to describe this pairing in a particular case $V_{\lambda}=V$ let us calculate the braiding of $V$ and $\Lambda^{p-1}(V)$ expressed via the basis $\left\{e^{i}\right\}$. Note, that we use the renormalized braiding $\bar{R}(2.34)$.

Proposition 13 We have

$$
\bar{R}_{V, V^{*}}: e_{i} \otimes e^{j} \longrightarrow e^{r} \otimes e_{s} \bar{Q}_{i r}^{j s}
$$

where in the given basis $\left\{e_{i}\right\}$ the operator $\bar{Q}$ satisfies the relation:

$$
\bar{R}_{j b}^{i a} \bar{Q}_{a l}^{b k}=\delta_{l}^{i} \delta_{j}^{k} \quad \Leftrightarrow \quad \bar{Q}_{j b}^{i a} \bar{R}_{a l}^{b k}=\delta_{l}^{i} \delta_{j}^{k} .
$$

Note, that if such $\bar{Q}$ exists then the corresponding braiding $\bar{R}$ is usually called invertible by column.

Proof. According to definition (2.42) for $e^{i}$ and (2.35) for the renormalized braiding we get (omitting the obvious signs of tensor product):

$$
e_{i} \otimes e^{j}=e_{i} e_{a_{2}} \ldots e_{a_{p}} v^{j a_{2} \ldots a_{p}} \stackrel{\bar{R}_{V, V \otimes(p-1)}}{\longrightarrow} e_{s_{1}} \ldots e_{s_{p}}( \pm q)^{\frac{1-p}{p}}\left(R_{p-1} \ldots R_{1}\right)^{s_{1} \ldots a_{2} \ldots a_{p}} v^{j a_{2} \ldots a_{p}} .
$$


Now from (2.28) it is easy to find

$$
( \pm q)^{\frac{1-p}{p}}\left(R_{p-1} \ldots R_{1}\right)_{i a_{2} \ldots a_{p}}^{s_{1} \ldots s_{p}} v^{j a_{2} \ldots a_{p}}=\bar{Q}_{i r}^{j s_{p}} v^{r s_{1} \ldots s_{p-1}}
$$

Substituting this into the previous relation and applying again the definition (2.42) of the dual basis we find result (2.47).

Now we introduce a pairing between $V$ and $V^{*}$ by putting

$$
\mathbf{e v}_{V}=\mathbf{e v}_{V} \circ R_{V, V^{*}}: \quad V \otimes V^{*} \rightarrow \mathbb{K}
$$

It is this operator which plays the central role in the next section where a categorical trace in the space End $(V)$ will be introduced.

Completing this Section let us discuss a meaning of the property that the morphism $\psi$ is coordinated with the braidings in the sense of (1.3). Let $V$ be the basic object of the category $\mathrm{SW}(\mathrm{V})$. Assuming the category to be rigid we can identify End $(V) \cong V \otimes \Lambda^{(p-1)}(V)$ (we consider the left morphisms space). Then the usual operator product $\mu:$ End $(V)^{\otimes 2} \rightarrow$ End $(V)$ is nothing but the pairing $\Lambda^{(p-1)}(V) \otimes V \rightarrow \mathbb{K}$. Moreover, the property

$$
R \mu_{12}=\mu_{23} R_{12} R_{23}, \quad R \mu_{23}=\mu_{12} R_{23} R_{12}, \quad R=R_{\operatorname{End}(V), \operatorname{End}(V)}
$$

which appears in numerous applications is satisfied. Let us note that these relations are not invariant w.r.t. a rescaling $R \rightarrow a R, a \neq 0$ while the condition (2.40) is.

Also remark that often a problem arises to check that a given map $\rho: U \rightarrow V, U, V \in \mathrm{Ob}(\mathcal{C})$ is a categorical morphism. Then by putting in (1.3) $f=\rho, g=$ id or $f=\rho, g=\rho$ we get necessary conditions very useful in practice.

\section{Additive-multiplicative functional and dimension}

In this section we find all possible additive and multiplicative (a-m) functionals on objects of Schur-Weyl category SW(V) and suggest a definition of the trace in $\operatorname{End}(V)$ as morphism (1.1) in the category such that the corresponding dimension is an a-m functional. As was pointed out in the Introduction, the requirement that the trace should be a morphism in general is not a trivial one. In particular, this requirement gives rise to the notion of quantum trace in the category of finite dimensional modules over a special class of quasitriangular Hopf algebras the so called ribbon Hopf algebras. In our approach the morphism property of trace can be obtained without any additional Hopf structure.

Let us recall that a mapping $f: \mathrm{Ob}(\mathrm{SW}(\mathrm{V})) \rightarrow \mathbb{K}$ will be called an additive-multiplicative functional (a-m functional) if it possesses the following property:

$$
\forall U, V \in \mathrm{Ob}(\mathrm{SW}(\mathrm{V})): \quad f(U \oplus V)=f(U)+f(V), \quad f(U \otimes V)=f(U) f(V) .
$$

Remark 14 Introducing the Grothendieck semiring of the category $S W(V)$ and making use of the fact that our category is semisimple (i.e. any its object can be decomposed into a direct sum of simple ones) we conclude that each a-m functional on the category is in fact defined on the Grothendieck semiring.

The fact that we are working with a Hecke symmetry of the rank $p$ leads to an important consequence for the set of a-m functionals. Namely we will show that the value of any a-m functional $f$ on a basic object $V_{\lambda}$ (and, hence, on an arbitrary object of SW(V)) is completely determined by $(p-1)$ numbers $5\left(V_{\left(1^{k}\right)}\right) k=1, \ldots, p-1$.

\footnotetext{
${ }^{12}$ Due to $(2.21)$ and reduction morphism $\psi 2.26$ we have to put for any a-m functional $f(\mathbb{K})=f\left(V_{\left(1^{p}\right)}\right)=1$.
} 
Proposition 15 Given an a-m functional $f$, construct a p-th order polynomial in a formal variable $t$ of the form:

$$
\phi(t)=t^{p}+f_{1} t^{p-1} \ldots+f_{p-1} t+1, \quad f_{k} \equiv f\left(V_{\left(1^{k}\right)}\right), \quad k=1, \ldots, p-1 .
$$

Let numbers $\left(-\alpha_{i}\right) \in \mathbb{K}$ be the roots of $\phi(t)$, that is $\phi\left(-\alpha_{i}\right)=0 i=1, \ldots, p$. Then for any basic object $V_{\lambda}$ of $S W(V)$ the following relation holds:

$$
f\left(V_{\lambda}\right)=s_{\lambda}\left(\alpha_{1}, \alpha_{2}, \ldots, \alpha_{p}\right)
$$

where $s_{\lambda}\left(x_{1}, \ldots, x_{p}\right)$ is the symmetric Schur function in $p$ variables ${ }^{[3}$ corresponding to the partition $\lambda$.

Proof. First of all let us prove that the numbers $f_{k}=f\left(V_{\left(1^{k}\right)}\right)$ do satisfy (3.3). In accordance with the definition of $\alpha_{i}$ we can write

$$
\phi(t)=\left(t+\alpha_{1}\right)\left(t+\alpha_{2}\right) \ldots\left(t+\alpha_{p}\right) .
$$

Therefore, as immediately follows from this relation, the coefficient $f_{k}$ is the $k$-th elementary symmetric function $e_{k}$ in variables $\alpha_{i}[\mathrm{M}]$ :

$$
f_{k}=\sum_{i_{1}<i_{2}<\ldots<i_{k}} \alpha_{i_{1}} \alpha_{i_{2}} \ldots \alpha_{i_{k}}=e_{k}\left(\alpha_{1}, \ldots, \alpha_{k}\right) .
$$

But since $s_{\left(1^{k}\right)}=e_{k}$ we conclude that the assertion of the proposition is valid for $f_{k}$.

The fact that the quantities $s_{\lambda}\left(\alpha_{1}, \alpha_{2}, \ldots, \alpha_{p}\right)$ can be considered as values of an a-m functional follows immediately from the properties of Schur functions and from Proposition 6 . In order to prove (3.3) we should only check that once the quantities $f\left(V_{\lambda}\right), \lambda=\left(1^{k}\right)$, are given then all quantities $f\left(V_{\mu}\right)$ for all other partitions are uniquely defined. This can be shown by induction in couples $(m, k)$ where $m$ is the number of columns in $\lambda$ and $k$ is the number of boxes in the last column. Namely, using the implication

$$
f\left(V_{\lambda} \otimes V_{\mu}\right)=f\left(V_{\lambda}\right) f\left(V_{\mu}\right)=c_{\lambda \mu}^{\nu} f\left(V_{\nu}\right) \Rightarrow s_{\lambda} s_{\mu}=c_{\lambda \mu}^{\nu} f\left(V_{\nu}\right)
$$

we can find the quantity $f\left(V_{\lambda}\right)$ for $\lambda$ corresponding to a given couple $(m, k)$ provided that all $f\left(V_{\mu}\right)$ where $\mu$ corresponds to the couples $(l, r)$ such that $l<m$ or $l=m, r<k$ are already known.

In what follows we will use this proposition for a proper definition of the categorical trace. It will be defined as such a morphism (1.1) that the corresponding dimension becomes an a-m functional. By virtue of the above proposition we should only find the quantities (3.3) for $\lambda=\left(1^{k}\right)$.

Describe now this construction in detail. Begin with the basic object $V$. Since the space $V$ is finite dimensional, we can identify $\operatorname{End}(V)$ and $V \otimes V^{*}$ in the usual way:

$$
\forall \mathcal{F} \in \operatorname{End}(V) \longleftrightarrow e_{i} \otimes F_{j}^{i} e^{j} \in V \otimes V^{*}
$$

$F_{j}^{i}$ being a matrix of $\mathcal{F}$ in the basis $\left\{e_{i}\right\}$. Define the mapping $\operatorname{tr}_{V}: \operatorname{End}(V) \rightarrow \mathbb{K}$ as the following composition of morphisms:

$$
\operatorname{tr}_{V}=\alpha \mathbf{e v}_{V} \circ \bar{R}_{V, V^{*}}
$$

Note, that $\operatorname{tr}_{V}$ is defined by the above relation up to an arbitrary nonzero factor $\alpha$ which will be specified later from the requirement that the corresponding dimension would be an a-m

\footnotetext{
${ }^{13}$ Let us recall, that due to $(2.21)$ all the partitions $\lambda$ labelling the objects of our category has the height not greater than $p$ therefore the right hand side of equation (3.3) is correctly defined for any object of SW(V).
} 
functional. In the fixed basis $\left\{e_{i}\right\}$ of $V$ one can write this mapping in an explicit form. Taking $\bar{R}_{V, V^{*}}$ from Proposition 13 we find:

$$
\forall \mathcal{F} \in \operatorname{End}(V): \quad e_{i} \rightarrow e_{j} F_{i}^{j} \Rightarrow \operatorname{tr}_{V}(\mathcal{F})=\alpha \operatorname{Tr}(F \cdot C)
$$

where the symbol Tr means the usual matrix trace and the $n \times n$ matrix $C$ is defined to be

$$
C_{j}^{i}:=\sum_{a=1}^{n} Q_{j a}^{i a}, \quad Q \equiv( \pm q)^{-\frac{1}{p}} \bar{Q}
$$

Here the matrix $\bar{Q}$ is taken from $(2.48)$ and the factor $( \pm q)^{\frac{1}{p}}$ is included into $\alpha$.

The matrix $C$ has the following useful properties GPS:

$$
\begin{aligned}
& R_{12} C_{1} C_{2}=C_{1} C_{2} R_{12} \\
& C_{\left\langle 1^{\prime}\right|}^{|1\rangle}=\frac{p_{q}}{q^{p}} u_{\left\langle 1^{\prime} 23 \ldots p\right|} v^{|123 \ldots p\rangle} \quad \Rightarrow \quad \operatorname{Tr} C=\frac{p_{q}}{q^{p}} . \\
& \operatorname{Tr}_{(2)} R_{12} C_{2}=\operatorname{id}_{(1)} .
\end{aligned}
$$

The $\operatorname{trace}_{\operatorname{tr}^{\otimes k}}$ in $\operatorname{End}\left(V^{\otimes k}\right)$ is defined (also up to an arbitrary factor $\beta_{(k)}$ ) as the mapping $V^{\otimes k} \otimes V^{* \otimes k} \rightarrow \mathbb{K}:$

$$
\operatorname{tr}_{V \otimes k}=\beta_{(k)} \operatorname{tr}_{V}^{(1)} \circ \operatorname{tr}_{V}^{(2)} \ldots \circ \operatorname{tr}_{V}^{(k)}
$$

where $\operatorname{tr}_{V}^{(i)}: V^{\otimes i} \otimes V^{* \otimes i} \rightarrow V^{\otimes(i-1)} \otimes V^{* \otimes(i-1)} \operatorname{reads}$

$$
\operatorname{tr}_{V}^{(i)}=\mathrm{id}^{\otimes(i-1)} \otimes \alpha^{-1} \operatorname{tr}_{V} \otimes \mathrm{id}^{\otimes(i-1)}
$$

For any basic object $V_{\lambda} \lambda \vdash m$ one can find the explicit form of the trace in $\operatorname{End}\left(V_{\lambda}\right) \cong \operatorname{End}\left(V_{\lambda}(i)\right)$ using definitions (2.11), (2.45) and property (3.6):

$$
\forall \mathcal{F} \in \operatorname{End}\left(V_{\lambda}(i)\right): \quad \operatorname{tr}_{V_{\lambda}}(\mathcal{F})=\beta_{(m)} \operatorname{Tr}_{(1 \ldots m)}\left(F \cdot C_{\lambda}\right)
$$

where $\operatorname{Tr}_{(1 \ldots m)}$ is the the usual trace in the matrix space $\operatorname{Mat}_{n \times n}^{\otimes m}(\mathbb{K})$ and the matrix $C_{\lambda}$ is of the form:

$$
C_{\lambda}=\mathcal{Y}_{i i}^{\lambda}\left(R_{1} \ldots R_{m-1}\right) C_{1} C_{2} \ldots C_{m}
$$

Remark 16 At the first sight the trace $\operatorname{tr}_{V_{\lambda}}$ depends on a concrete embedding $V_{\lambda} \rightarrow V_{\lambda}(i)$. But in fact our definition is invariant w.r.t. different embeddings of $V_{\lambda}$ into $V^{\otimes m}$. Indeed, as was already noticed in the previous section, any primitive idempotents $Y_{i i}^{\lambda}$ of the Hecke algebra $H_{m}$ can be transformed into another idempotent $Y_{j j}^{\lambda}$ (with the same $\lambda$ ) with the help of an invertible element $X(i, j \mid \lambda)$ from $H_{m}$. The image $\mathcal{X}=\rho_{R}(X(i, j \mid \lambda))$ of the element $X(i, j \mid \lambda)$ under the representation $\rho_{R}$ (2.1) represents a first kind morphism of $S W(V)$ and according to (3.6) the string of matrices $C_{1} \ldots C_{m}$ in (3.11) commute with $\mathcal{X}$. Therefore trace (3.10) is actually independent of the index $i$ labelling the concrete embedding $V_{\lambda} \rightarrow V_{\lambda}(i)$.

Find now the conditions on arbitrary factors $\alpha$ and $\beta_{(k)}$ which would guarantee the dimensions defined via the above trace to be an a-m functional.

Let us note that although the dimension defined by the usual trace is an a-m functional such a trace is not a morphism of the category if the braiding differs from the flip 4 . This is the reason why we should modify the usual trace by means of the matrix $C$ (and all its extensions $C_{\lambda}$ ) multiplied by a proper factor $\alpha(3.5)$.

\footnotetext{
${ }^{14}$ For example, if our category $\mathrm{SW}(\mathrm{V})$ is supplied with the comodule structure over the RTT Hopf algebra (2.36) then the usual trace is not compatible with the comodule structure.
} 
The fact that the matrix $C$ extends to $\operatorname{End}\left(V^{\otimes m}\right)$ in a "group like" way

$$
C \rightarrow C^{\otimes m}
$$

ensures the conservation of the additive and multiplicative structure of the functional (3.9) restricted on the identity operator (what is just categorical dimension) iff we put

$$
\beta_{(k)}=\alpha^{k} \text {. }
$$

So, if we want the trace $\operatorname{tr}_{V_{\lambda}}$ to be a morphism and giving rise to an a-m functional we have the only free parameter $\alpha$ at our disposal. This parameter (a normalization of the trace) can be found from the following condition $\operatorname{dim}_{q} V_{\left(1^{p}\right)}=1$ (see footnote 12). The following lemma plays the key role in finding such a normalization (the relation presented below was found in another but equivalent form in [H]).

Lemma 17 For any Hecke symmetry of the rank $p$ the following relation holds:

$$
\operatorname{Tr}_{(1 \ldots k)}\left(\mathcal{A}^{(k)} C_{1} \ldots C_{k}\right)=q^{-p k} \frac{p_{q} !}{k_{q} !(p-k)_{q} !}, \quad 1 \leq k \leq p
$$

where the matrix $\mathcal{A}^{(k)}$ is the image of $k$-th order antisymmetrizer $A^{(k)}$ under the representation $\rho_{R}$ (2.1).

Proof. For an arbitrary $m \in \mathbb{Z}$ introduce the auxiliary notation

$$
\mathcal{R}_{i}(m):=R_{i}-\frac{q^{m}}{m_{q}}
$$

where $m_{q}$ is defined in (1.9). With this notation the matrix $\mathcal{A}^{(m)}\left(R_{1}, \ldots, R_{m-1}\right)$ can be presented in the form:

$$
\mathcal{A}^{(m)}={\frac{(-1)}{m_{q}}}^{m-1} \mathcal{A}^{(m-1)} \mathcal{R}_{m-1}(m-1) \mathcal{R}_{m-2}(m-2) \ldots \mathcal{R}_{1}(1), \quad \mathcal{A}^{(1)}:=\mathrm{id} .
$$

For the sake of shortness we introduce the notation:

$$
\operatorname{Tr}_{q(1 \ldots k)} X:=\operatorname{Tr}_{(1 \ldots k)}\left(X C_{1} \ldots C_{k}\right) .
$$

Now we prove (3.13) by induction. For $k=1$ we get with the help of (3.7)

$$
\operatorname{Tr} C=q^{-p} p_{q}
$$

which gives the base of induction. Suppose that the assertion of the lemma is valid up to some integer $k<p$. Then using the simple relations

$$
\begin{aligned}
& \operatorname{Tr}_{q(k+1)} \mathcal{R}_{k}(k)=-q^{-p} \frac{(p-k)_{q}}{k_{q}} \operatorname{id}_{(k)} \\
& \mathcal{A}^{(k)} \mathcal{R}_{k-1}(k-1) \ldots \mathcal{R}_{1}(1)=(-1)^{k-1} k_{q} \mathcal{A}^{(k)} .
\end{aligned}
$$

one can complete the induction

$$
\begin{aligned}
\operatorname{Tr}_{q(1 \ldots k+1)} \mathcal{A}^{(k+1)} & =\frac{(-1)^{k}}{(k+1)_{q}} \operatorname{Tr}_{q(1 \ldots k+1)}\left(\mathcal{A}^{(k)} \mathcal{R}_{k}(k) \ldots \mathcal{R}_{1}(1)\right) \\
& =\frac{(-1)^{k}}{(k+1)_{q}} \operatorname{Tr}_{q(1 \ldots k)}\left(\mathcal{A}^{(k)}\left[\operatorname{Tr}_{q(k+1)} \mathcal{R}_{k}(k)\right] \mathcal{R}_{k-1}(k-1) \ldots \mathcal{R}_{1}(1)\right) \\
& =(-1)^{k+1} q^{-p} \frac{(p-k)_{q}}{k_{q}(k+1)_{q}} \operatorname{Tr}_{q(1 \ldots k)}\left(\mathcal{A}^{(k)} \mathcal{R}_{k-1}(k-1) \ldots \mathcal{R}_{1}(1)\right) \\
& =q^{-p} \frac{(p-k)_{q}}{(k+1)_{q}} \operatorname{Tr}_{q(1 \ldots k)} \mathcal{A}^{(k)}=\frac{q^{-p(k+1)} p_{q} !}{(k+1)_{q} !(p-k-1)_{q} !} .
\end{aligned}
$$


This lemma implies that if we take $\alpha=q^{p}$ (which is equivalent to simple renormalization $C \rightarrow q^{p} C$ in all formulas for the trace) then we get $\operatorname{dim}_{q} V_{\left(1^{p}\right)}=1$ and the categorical dimension related to the trace becomes an a-m functional well defined on objects of our category and hence on the corresponding Grothendieck semiring.

Let us summarize the above consideration in the following proposition.

Proposition 18 For any basic object $V_{\lambda}, \lambda \vdash m$, of the category $S W(V)$ define the trace $\operatorname{tr}_{V_{\lambda}}$ as a morphism $\operatorname{End}\left(V_{\lambda}\right) \rightarrow \mathbb{K}$ of the form:

$$
\forall \mathcal{F} \in \operatorname{End}\left(V_{\lambda}\right): \quad \operatorname{tr}_{V_{\lambda}}(\mathcal{F})=q^{p m} \operatorname{Tr}_{(1 \ldots m)}\left(F \cdot C_{\lambda}\right) .
$$

Then such a trace is a morphism in the category $S W(V)$ and the corresponding categorical dimension is an a-m functional. The dimension of any basic object $V_{\lambda}$ is as follows:

$$
\operatorname{dim}_{q} V_{\lambda}:=\operatorname{tr}_{V_{\lambda}}(\mathrm{id})=s_{\lambda}\left(q^{p-1}, q^{p-3}, \ldots, q^{1-p}\right)
$$

$s_{\lambda}$ being the Schur symmetric function (polynomial) in $p$ variables.

Proof. To prove (3.14) we use Proposition 15 and Lemma 17. By virtue of the trace definition one gets from (3.13):

$$
\operatorname{dim}_{q} V_{\left(1^{k}\right)}=q^{p k} \operatorname{Tr}_{q(1 \ldots k)} \mathcal{A}^{(k)}=\frac{p_{q} !}{k_{q} !(p-k)_{q} !} \equiv f_{k}
$$

Therefore due to Proposition 15 we should find the roots of the polynomial $\phi(t)$ (3.2) with the above $f_{k}$. But as is well known the generating function for such $f_{k}$ (which are $q$-binomial coefficients) is as follows

$$
E_{q}(t):=\prod_{k=0}^{p-1}\left(q^{2 k+1-p}+t\right) \equiv \phi(t) .
$$

Therefore the roots of the polynomial $\phi(t)$ with the coefficients $f_{k}=\operatorname{dim}_{q} V_{\left(1^{k}\right)}$ are the numbers $\left(-q^{2 k+1-p}\right), k=0,1, \ldots, p-1$ and result (3.14) follows now from Proposition 15 .

Remark 19 We want to complete the paper with the following observation concerning the Koszul complexes considered in [G]. As was shown there, the Poincaré series $P_{ \pm}(t)$

$$
P_{ \pm}(t):=\sum_{l} \operatorname{dim} \Lambda_{ \pm}^{l} t^{l}
$$

of "symmetric" and "skewsymmetric" algebras $\Lambda_{ \pm}(V)$ (see (1.10)) satisfy the relation

$$
P_{+}(t) P(-t)=1 \text {. }
$$

We would like to point out that if we replace the usual dimensions in formula (3.15) by categorical ones relation (3.15) will be still valid. Moreover, the same is true if we replace the dimensions by the values of any a-m functional. It is not surprising since this fact reflects the well known relation between elementary and complete symmetric functions. And any a-m functional is nothing but a specialization of symmetric functions.

Thus, without constructing any deformed Koszul complex we can obtain some numerical characteristics of quantum objects merely replacing the usual dimensions by their q-analogs (if we disregard the property of the quantum differential to be a morphism of the category). 


\section{References}

[B] Banica, A reconstruction result for $R$-matrix quantization of $S U(n)$, QA/9806063.

[BW] J.W.Barrett and B.W.Westbery, Spherical Categories, Advances in Mathematics 143 (1999), pp 357-375.

[CP] V.Chari and A. Pressley, A guide to Quantum Groups, Cambrige University Press, 1994.

[DM] P.Deligne and J.S.Miln, Tannakian Categories in: P.Deligne, J.S.Miln, A.Ogus, Shing Kuang-yen Hodges Cycles, Motives, and Shimura Varieties, Lect. Notes in Math. 900, Berlin, Heidelberg, NY: Springer-Verlag, 1982, pp. 101-228.

[DJ] R. Dipper and G.James, Representations of Hecke algebras of General Linear Groups. Proc. London Math. Soc., 52(3) (1986), pp 20-52.

R. Dipper and G.James, Block and Idempotents of Hecke algebras of General Linear Groups, Proc. London Math. Soc., 54(3) (1987), pp 57-82.

[DP] A.Dold and D.Puppe, Duality, trace and transfer (Russian), Topology (Moscow 1979), Trudy Mat. Ins. Steklov 154 (154), pp 81-97.

[DL] M.Dubois-Violette and G.Launer, The quantum group of a nondegenerated bilinear form, Phys.Lett. B245 (1990), pp 175-177.

[FRT] L.D.Faddeev, N.Yu.Reshetikhin and L.A.Takhtajan, Algebra i Analiz 1 (1989) 178 206 (in Russian); English translation in: Leningrad Math. J. 1 (1990), pp 193-226.

[Go] M.D.Gould, Quantum Groups and Diagonalization of the Braid Generator, Lett. Math. Phys. 24 (1992) pp. 183 - 196.

[G] D.Gurevich, Algebraic aspects of the quantum Yang-Baxter equation, Leningrad Math. J. 2 (1991), pp. 801-828.

[GM] D.Gurevich and Z.Mriss, Schur-Weyl Categories and Non-quasiclassical Weyl Type Formula, Hopf algebras and quantum groups (Brussels, 1998) Lec. Notes. in Pure and Appl. Math. 209, Dekker, NY 2000, pp 131-158.

[GPS] D.Gurevich, P.Pyatov and P.Saponov, Hecke Symmetries and Characteristic Relations on Reflection Equation Algebra, Lett. Mat. Phys. 41 (1997) pp. 255-264.

[HIOPT] L.K.Hadjiivanov, A.P.Isaev, O.V.Ogievetsky, P.N.Pyatov and I.T.Todorov, Hecke algebraic properties of dynamical $R$-matrices. Application to related quantum matrix algebras, q-alg/9712026

[H] Phung Ho Hai On matrix quantum groups of type $A_{n}$, Int. J. Math. 11 (2000), pp. $1115-1146$.

[KW] D.Kazhdan, H.Wenzl, Reconstructing Monoidal Categories, Adv. in Soviet. Math. 16, part 2 (1993), pp. 111-136.

[M] I.G.Macdonald, Symmetric functions and Hall Polynomials, Claredon Press, Oxford, 1979.

[McL1] S.MacLane, Natural associativity and commutativity, Rice Univ. Stud. 49 (1963), pp. $28-46$. 
[McL2] S.MacLane, Categories for the Working Mathematician, Graduate Texts in Mathematics, Springer Verlag, Berlin 1971.

[OP] O.Ogievetsky and P.Pyatov, Lecture on Hecke algebra, based on lectures at the International School "Symmetries and Integrable systems", Dubna, 8-11 June, 1999. JINR Publ. Dept., 2000.

[T] V.G.Turaev, Quantum invariants of Knots and 3-Manifolds, Walter de Gruyter, Berlin, NY 1994. 